\title{
Information and Volatility
}

\author{
Dirk Bergemann ${ }^{\mathrm{a}}$, Tibor Heumann ${ }^{\mathrm{b}}$, Stephen Morris ${ }^{\mathrm{c}}$ \\ ${ }^{a}$ Department of Economics, Yale University, New Haven, CT 06520, U.S.A. dirk.bergemann@yale.edu. \\ ${ }^{b}$ Department of Economics, Yale University, New Haven, CT 06520, U.S.A. tibor.heumann@yale.edu. \\ ${ }^{c}$ Department of Economics, Princeton University, Princeton, NJ 08544,U.S.A. smorris@princeton.edu.
}

\begin{abstract}
In an economy of interacting agents with both idiosyncratic and aggregate shocks, we examine how the structure of private information influences aggregate volatility. The maximal aggregate volatility is attained in a noise free information structure in which the agents confound idiosyncratic and aggregate shocks, and display excess response to the aggregate shocks, as in Lucas [14]. For any given variance of aggregate shocks, the upper bound on aggregate volatility is linearly increasing in the variance of the idiosyncratic shocks. Our results hold in a setting of symmetric agents with linear best responses and normal uncertainty. We establish our results by providing a characterization of the set of all joint distributions over actions and states that can arise in equilibrium under any information structure. This tractable characterization, extending results in Bergemann and Morris [8], can be used to address a wide variety of questions linking information with the statistical moments of the economy.
\end{abstract}

Keywords: Idiosyncratic Shocks, Aggregate Shocks, Volatility, Confounding Information, Moment Restrictions, Bayes Correlated Equilibrium.

JEL Classification: C72, C73, D43, D83.

\footnotetext{
We acknowledge financial support through NSF Grants SES 0851200 and ICES 1215808. We thank the Special Issue Editors, Alessandro Pavan and Xavier Vives, and the referees for many thoughtful comments. We are grateful to our discussant, Marios Angeletos, for many insightful suggestions. We thank the audience at the Workshop on Information, Competition and Market Frictions, Barcelona 2013, for helpful comments on an earlier version that was entitled "Information, Interdependence, and Interaction: Where Does Volatility Come From?"
} 


\section{Introduction}

Consider an economy of interacting agents, each of whom picks an action. Agents are subject to idiosyncratic and aggregate shocks. A classical economic question in this environment is to ask how aggregate and idiosyncratic shocks map into "aggregate volatility" - the variance of the average action. Versions of this question arise in many different economic contexts. In particular, a central question in macroeconomics is how aggregate and individual productivity shocks translate into variation in GDP. Another classical question is when and how asymmetric information can influence this mapping, and in particular exacerbate aggregate volatility. A difficulty addressing this question is that the answer depends on the nature of the asymmetric information, something that is not easily observed. Thus results may be sensitive to the exact information structure assumed.

This paper considers a very simple stylized economy where we can completely characterize what can happen for all information structures. In particular, we consider a setting with a continuum of agents with linear best responses that depend on the average action of others and idiosyncratic and aggregate shocks. We assume that shocks, actions and signals are symmetrically normally distributed across agents, maintaining symmetry and normality of the information structure. Our sharp characterization of what can happen across all information structures in this symmetric normal class can be used to address many economic questions of interest. In particular, we can study the two classical questions described above, providing an upper bound on aggregate volatility as a function of fundamentals and identifying the critical information structures that give rise to maximal volatility.

The information structure that maximizes aggregate volatility turns out to be "noise free": each agent observes a one-dimensional signal which is a deterministic function of his idiosyncratic and the aggregate shock. While there is no noise in such signals, they are imperfect because they leave the agent uncertain about the size of the idiosyncratic and aggregate shocks. The shocks are confounded in the agent's signal. Aggregate volatility is highest when signals overweigh the aggregate shock relative to the idiosyncratic shock. In this case, agents who want to tailor their

actions to their idiosyncratic shocks have no choice but to overweigh the aggregate shock, generating aggregate volatility. We show how maximum aggregate volatility increases linearly in the variance of the idiosyncratic shocks even if the variance of aggregate shocks is held constant. The critical noise free signal generating the maximal aggregate volatility puts proportionately more weight on the aggregate shock of constant variance as the variance of the idiosyncratic shock becomes larger. 
These noise free information structures are also critical for many questions of interest, including for dispersion (the variance of individual actions around the mean action) and individual volatility (the variance of individual actions, which is equal to the sum of aggregate volatility and dispersion). But different noise free information structures maximize these variables. Thus dispersion is highest when signals overweigh the idiosyncratic shock relative to the aggregate shock. In this case, agents who want to tailor their actions to their aggregate shocks have no choice but to overweigh the idiosyncratic shock, generating dispersion.

The fact that confounding shocks can lead to overreaction has been long recognized, notably by Lucas [14] and more recently by Hellwig and Venkateswaran [11] and Venkateswaran [21]. Our contribution is to highlight that, in this setting with idiosyncratic and aggregate shocks, noise free confounding information structures are extremal and provide global bounds on how much volatility can arise via the information structure. The intuition for the bounding result is simple and comes in two parts. First, suppose that agents observed a one-dimensional signal that was a linear function of the idiosyncratic shock, the aggregate shock and a noise term which may be correlated across agents. Equilibrium actions must be linear in the signal. The impact of the noise in the signal must be to dampen the response of agents to the signal and thus to both the idiosyncratic and aggregate shocks. Thus among one-dimensional symmetric information structures, noise free information structures generate the most volatility. Second, imagine any other, perhaps multidimensional and perhaps noisy, symmetric information structure. By symmetry, each agent's equilibrium action choice (assumed to be one-dimensional) can be expressed as a linear function of the idiosyncratic shock, the aggregate shock and a noise term which may be correlated across agents. Now we can replace the original information structure by the one-dimensional one where each agent observes a signal which is linear in the equilibrium action he would have chosen under the old information structure. Equilibrium in this new information structure will now generate the same outcomes as the equilibrium with the richer information structure. Thus it is enough to study one-dimensional information structures where signals are a linear function of the idiosyncratic shock, the aggregate shock and a noise term which may be correlated across agents.

We provide a complete characterization of the symmetric equilibrium distributions of individual actions, aggregate actions, idiosyncratic shocks and aggregate shocks that can arise for a given distribution of payoff shocks across all possible information structures. The direct characterization of all feasible equilibrium distributions - independent of the information structure - makes use of a solution concept, Bayes correlated equilibria, introduced in earlier work by two of us, Bergemann 
and Morris [8], [8]. Namely, the set of Bayes correlated equilibrium distributions is equal to the set of Bayes Nash equilibrium distributions under all possible information structures. And in particular, the boundary of the set of Bayes correlated, and hence Bayes Nash equilibria, can be written as the (Bayes Nash) equilibrium of some noise free and one-dimensional information structure. The resulting bounds of the aggregate volatility are independent of the assumption of normality of the shocks, and only depend on the variance of the idiosyncratic and the aggregate shocks.

While noise free information structures generate maximal aggregate volatility with both idiosyncratic and aggregate shocks, this is not longer true if there are only idiosyncratic shocks. In this case, if each agent responded to his idiosyncratic shock only, there would be no aggregate volatility by the law of large numbers. On the other hand, if each agent had no information about his idiosyncratic shock, his action would be constant and there would again be no aggregate volatility. The information structure which maximizes aggregate volatility would be one where each agent observes his idiosyncratic shock with an intermediate level of noise, and where the noise in agents' signals is perfectly correlated. Angeletos and La'O [4] have analyzed the role of such aggregate shocks to beliefs about purely idiosyncratic uncertainty in a macroeconomic model, describing them as "sentiment" shocks. How can we relate this finding that - with only idiosyncratic shocks - adding noise maximizes aggregate volatility to our finding that - adding aggregate shocks - noise can only decrease aggregate volatility? We can reconcile the results by considering what happens if we let the variance of aggregate shocks decline towards zero in our model. In this case, our results show that the information structure that maximizes aggregate volatility is a noise free information structure where the signal puts a larger and larger weight on the aggregate shock and a smaller and smaller weight on the idiosyncratic shock. The agent - in order to respond to the idiosyncratic shock at all - must put a larger and larger weight on the signal. The total sensitivity to the aggregate shock (multiplying the weight on the aggregate shock in the signal with the weight on the signal in the equilibrium strategy) converges to a constant as the aggregate shock disappears. In the limit the dependence on the aggregate payoff shock becomes dependence on a common, payoff irrelevant, noise term, i.e., the sentiment shock. Thus this paper highlights a tight connection between noise free, but confounding information structures and sentiment shocks.

The results described thus far hold independently of whether the weight on the average action, $r$, is negative (the strategic substitutes case), zero (the purely decision theoretic case) or positive (the strategic complementarities case). A striking property of our characterization of Bayes correlated equilibria - i.e., what can happen in all symmetric information structures - is that the set of feasible 
correlations between individual and average actions and individual and aggregate shocks is independent of $r$ and determined only by statistical constraints. There are three degrees of freedom in describing the correlation structure: the correlation between each agent's action and his individual shock (the sum of the aggregate shock and his idiosyncratic shock), the correlation between any two agents' actions, and the correlation between any agent's action and any other agent's individual shock. On the other hand, once one pins down the correlation structure, the mean and variance of individual actions are pinned down. The best response parameter $r$ describes how to translate correlation structures into first and second moments. Thus there is a three-dimensional class of Bayes correlated equilibria which is extremely tractable.

While we can restrict attention to one-dimensional information structures in deriving bounds on volatility, we may want to assume that agents have access to particular class of (possibly multidimensional) signals. In general, this will impose restrictions on the set of Bayes Nash equilibria and the upper bounds on volatility cannot necessarily be obtained. For example, in Angeletos and La'O [4], each agent knows his individual payoff shock but remains uncertain about the payoff shock of the other agents. In Angeletos and Pavan [6] the agents have access to a rich three-dimensional class of normal signals, and even though the set of Bayes correlated equilibria is also three-dimensional, the information structures of Angeletos and Pavan [6] do not give rise to maximal volatility.

This paper is an application of a general approach to analyzing equilibrium behavior of agents for a given description of the fundamentals for all possible information structures. In Bergemann and Morris [9], we consider this problem in a canonical game theoretic setting and show that a general version of Bayes correlated equilibrium characterizes the set of outcomes that could arise in any Bayes Nash equilibrium of an incomplete information game where agents may have access to more information beyond the given common prior over the fundamentals. In Bergemann and Morris [8] we pursue this argument in detail and characterize the set of Bayes correlated equilibria in the class of games with quadratic payoffs and normally distributed uncertainty, but there we restrict our attention to the special environment with aggregate shocks only. By providing a complete analysis of the impact of information in a simple linear quadratic model with both idiosyncratic and aggregate payoff shocks, we can connect with richer macroeconomic and other applied models that work with parameterized classes of information structures.

The remainder of the paper is organized as follows. Section 2 introduces the model. Section 3 introduces the noise free information structures and analyzes the Bayes Nash equilibrium behavior for this class of information structures. Section 4 derives the maximal volatility and dispersion in 
a benchmark model without strategic interaction. We identify the specific noise free information structure that attains the maximal volatility, and thus establish the link between information and volatility. Section 5 examines how the strategic interaction affects how the information structure impacts the statistical moments of the economy. In Section 6, we introduce symmetric Bayes correlated equilibria and establish an equivalence between the set of Bayes correlated equilibria and the set of Bayes Nash equilibria under any symmetric normal information structure. Section 7 then considers some particular information structures that have been studied, and highlights the subtle restrictions that such modelling choices imposes on the equilibrium behavior. Section 8 indicates how the present analysis extends beyond aggregate interaction to allow for richer, network like, interaction structures. Finally, we apply the currents insights to the related literature on information sharing and obtain optimal information sharing rules that differ and improve upon those appearing in the literature. Section 9 constitutes the appendix and contains most of the proofs.

\section{Model}

We consider a continuum of agents, with mass normalized to 1 . Agent $i \in[0,1]$ chooses an action $a_{i} \in \mathbb{R}$ and is assumed to have a quadratic payoff function,

$$
u_{i}: \mathbb{R}^{3} \rightarrow \mathbb{R}
$$

which is function of his action $a_{i}$, the mean action taken by all agents, $A$,

$$
A \triangleq \int_{j} a_{j} d j
$$

and the individual payoff shock, $\theta_{i} \in \mathbb{R}$, thus $u_{i}\left(a_{i}, A, \theta_{i}\right)$.

We assume that the individual payoff shock $\theta_{i}$ is given by the sum of an aggregate shock $\bar{\theta}$ and an idiosyncratic shock $\Delta \theta_{i}$ :

$$
\theta_{i}=\bar{\theta}+\Delta \theta_{i}
$$

The aggregate shock $\bar{\theta}$ is common to all agents and the idiosyncratic shock $\Delta \theta_{i}$ is identically and independently distributed across agents, as well as independent of the aggregate shock. Each component of the payoff shock $\theta_{i}$ is normally distributed. The payoff environment is thus completely described by the pair $\left(\bar{\theta}, \Delta \theta_{i}\right)$ of random variables,

$$
\left(\begin{array}{c}
\bar{\theta} \\
\Delta \theta_{i}
\end{array}\right) \sim \mathcal{N}\left(\left(\begin{array}{c}
\mu_{\bar{\theta}} \\
0
\end{array}\right),\left(\begin{array}{cc}
\sigma_{\bar{\theta}}^{2} & 0 \\
0 & \sigma_{\Delta \theta_{i}}^{2}
\end{array}\right)\right),
$$


which form the commonly known common prior. The sample average of the idiosyncratic shocks across all agents always equals zero. We denote the sample average across the entire population, that is across all $i$, as $\mathbb{E}_{i}[\cdot]$, and so $\mathbb{E}_{i}\left[\Delta \theta_{i}\right]=0$. The aggregate shock can be interpreted as the sample mean or average payoff shock, and so $\bar{\theta}=\mathbb{E}_{i}\left[\theta_{i}\right]$.

Given the independence and the symmetry of the idiosyncratic shock $\Delta \theta_{i}$ across agents, the variance of the individual payoff shock $\theta_{i}$ can be expressed in terms of the variance of the sum of the idiosyncratic and the aggregate shock:

$$
\sigma_{\theta}^{2} \triangleq \sigma_{\bar{\theta}}^{2}+\sigma_{\Delta \theta_{i}}^{2}
$$

The correlation (coefficient) $\rho_{\theta \theta}$ between the payoff shocks of any two agents $i$ and $j, \theta_{i}$ and $\theta_{j}$ is given by,

$$
\rho_{\theta \theta}=\frac{\sigma_{\bar{\theta}}^{2}}{\sigma_{\bar{\theta}}^{2}+\sigma_{\Delta \theta_{i}}^{2}},
$$

and the covariance between individual payoff shocks $\theta_{i}$ and $\theta_{j}$ equals the covariance between the individual shock $\theta_{i}$ and the aggregate shock $\bar{\theta}$. Thus, the correlation coefficient $\rho_{\theta \theta}$ represents a measure of the "commonality" of the shocks across agents. Separately, from the perspective of a given agent $i$, the correlation coefficient $\rho_{\theta \theta}$ measures the relative contribution of the aggregate shock $\bar{\theta}$ to the individual shock of agent $i, \theta_{i}=\bar{\theta}+\Delta \theta_{i}$.

Given the quadratic property of the payoff function, each agent $i$ has a linear best response function:

$$
a_{i}=r \mathbb{E}\left[A \mid \mathcal{I}_{i}\right]+\mathbb{E}\left[\theta_{i} \mid \mathcal{I}_{i}\right]
$$

where $\mathbb{E}\left[\cdot \mid \mathcal{I}_{i}\right]$ is the expectation conditional on the information $\mathcal{I}_{i}$ that agent $i$ has prior to taking an action $a_{i}$. The parameter $r \in \mathbb{R}$ of the best response function represents the strategic interaction among the agents. If $r<0$, then we have a game of strategic substitutes, if $r>0$, then we have a game of strategic complements.

For future reference, we briefly describe the Nash equilibrium under complete information. If each agent observes the idiosyncratic and the aggregate shock separately, and hence receives the two-dimensional signal $\left(\Delta \theta_{i}, \bar{\theta}\right)$, then the linear best response function gives rise the symmetric Nash equilibrium strategy:

$$
a_{i}\left(\Delta \theta_{i}, \bar{\theta}\right) \triangleq \Delta \theta_{i}+\frac{\bar{\theta}}{1-r}
$$

In the complete information equilibrium, each agent assigns weight 1 to the idiosyncratic shock $\Delta \theta_{i}$, and weight $1 /(1-r)$ to the aggregate shock $\bar{\theta}$. We assume that the interaction parameter $r$ is 
bounded above, or $r \in(-\infty, 1)$, which guarantees that there is a unique interior Nash equilibrium. 1

The present model of a continuum of players with quadratic payoffs and normally distributed idiosyncratic and aggregate shocks was first proposed by Vives [23] to analyze information sharing among agents with private, but noisy, information about the fundamentals. While the focus of the present paper is rather different, we shall briefly indicate in the conclusion how our approach also yields new insights to the large literature on information sharing. In the information sharing literature, the aggregate and the idiosyncratic shock, are often referred to as the common value and the private value component of the payoff shock $\theta_{i}$.

\section{Noise Free Bayes Nash Equilibrium}

We begin the analysis by considering a class of noise free information structures and then derive the Bayes Nash equilibrium behavior under these one-dimensional information structures. We consider the following one-dimensional class of signals:

$$
s_{i} \triangleq \lambda \Delta \theta_{i}+(1-\lambda) \bar{\theta}
$$

where the linear composition of the signal $s_{i}$ is determined by the parameter $\lambda \in[0,1]$. We restrict attention to symmetric information structures (across agents), all agents have the same parameter $\lambda$, and hence simply refer to the noise free information structure $\lambda$. In the present section, we consider the case of idiosyncratic and aggregate shocks, thus $\rho_{\theta \theta} \in(0,1)$, and we discuss the limit cases of pure idiosyncratic and pure aggregate shocks, thus $\rho_{\theta \theta} \in\{0,1\}$, in the next section.

The information structure $\lambda$ is noise free in the sense that every signal $s_{i}$ is a linear combination of the idiosyncratic and the aggregate shock, $\Delta \theta_{i}$ and $\bar{\theta}$, and no extraneous noise or error term enters the signal of each agent. Nonetheless, since the signal $s_{i}$ combines the idiosyncratic and the

\footnotetext{
${ }^{1}$ The best response (4) normalizes the weight on the payoff shock $\theta_{i}$ to 1 and assigns the weight $r$ to the average action $A$. A subset of us adopted this normalization, Bergemann and Morris [8], and we can directly contrast the results of the pure aggregate shock model there with the model of idiosyncratic and aggregate shocks here. An alternative normalization would assign weights $1-r$ to the payoff shock and $r$ to the average action, as in Morris and Shin [16] or Angeletos and Pavan [5]. With pure aggregate shocks, the later normalization has the advantage that under complete information, the equilibrium volatility of the individual or the aggregate action is independent of $r$, whereas in the present model $r$ scales the volatility. Yet, in a general model with idiosyncratic and aggregate shocks even the complete information volatility is affected by $r$ (since then $\left.a_{i}\left(\Delta \theta_{i}, \bar{\theta}\right)=(1-r) \Delta \theta_{i}+\bar{\theta}\right)$ and hence this normalization would also fail to preserve the invariance property for the individual volatility.
} 
aggregate shock, each signal $s_{i}$ leaves agent $i$ with residual uncertainty about the contribution of the idiosyncratic and aggregate shock respectively. Moreover, unless the weight $\lambda$ in the information structure exactly mirrors the composition of the payoff shock, $\theta_{i}=\bar{\theta}+\Delta \theta_{i}$, and hence exactly equals $1 / 2$, agent $i$ still faces residual uncertainty about his payoff shock $\theta_{i}$. Thus, the signal confounds the two sources of payoff uncertainty.

Given the information structure $\lambda$, we can compute the conditional expectation of agent $i$ given the signal $s_{i}$ about the idiosyncratic shock $\Delta \theta_{i}:^{2}$

$$
\mathbb{E}\left[\Delta \theta_{i} \mid s_{i}\right]=\frac{\operatorname{cov}\left(\Delta \theta_{i}, s_{i}\right)}{\operatorname{var}\left(s_{i}\right)}=\frac{\left(1-\rho_{\theta \theta}\right) \lambda}{\rho_{\theta \theta}(1-\lambda)^{2}+\left(1-\rho_{\theta \theta}\right) \lambda^{2}} s_{i},
$$

the aggregate shock $\bar{\theta}$ :

$$
\mathbb{E}\left[\bar{\theta} \mid s_{i}\right]=\frac{\operatorname{cov}\left(\bar{\theta}, s_{i}\right)}{\operatorname{var}\left(s_{i}\right)}=\frac{\rho_{\theta \theta}(1-\lambda)}{\rho_{\theta \theta}(1-\lambda)^{2}+\left(1-\rho_{\theta \theta}\right) \lambda^{2}} s_{i},
$$

and the payoff shock $\theta_{i}$ of agent $i$ :

$$
\mathbb{E}\left[\theta_{i} \mid s_{i}\right]=\frac{\operatorname{cov}\left(\theta_{i}, s_{i}\right)}{\operatorname{var}\left(s_{i}\right)}=\frac{\rho_{\theta \theta}(1-\lambda)+\left(1-\rho_{\theta \theta}\right) \lambda}{\rho_{\theta \theta}(1-\lambda)^{2}+\left(1-\rho_{\theta \theta}\right) \lambda^{2}} s_{i} .
$$

For the above conditional expectations, it is only the relative size of idiosyncratic and aggregate shocks, thus $\rho_{\theta \theta}$, that matters rather than the level of the shocks, the variance $\sigma_{\Delta \theta_{i}}^{2}$ and $\sigma_{\bar{\theta}}^{2}$.

A few noise free information structures are of particular interest. If $\lambda=1 / 2$, then each agent knows his own payoff shock $\theta_{i}$, as (9) reduces to $\mathbb{E}\left[\theta_{i} \mid s_{i}\right]=2 s_{i}=\Delta \theta_{i}+\bar{\theta}$, but remains uncertain about the exact value of the idiosyncratic and aggregate shock. Similarly, if $\lambda=0$, then the aggregate shock is known with certainty by each agent, as $\mathbb{E}\left[\bar{\theta} \mid s_{i}\right]=s_{i}=\bar{\theta}$, but there remains residual uncertainty about the idiosyncratic shock $\Delta \theta_{i}$ and a fortiori about the payoff shock $\theta_{i}$. Likewise, if $\lambda=1$, then the idiosyncratic shock is known with certainty, as $\mathbb{E}\left[\Delta \theta_{i} \mid s_{i}\right]=s_{i}=\Delta \theta_{i}$, but there remains residual uncertainty about the aggregate shock $\bar{\theta}$ and a fortiori about the payoff shock $\theta_{i}$.

We record the standard solution concept for games of incomplete information.

\footnotetext{
${ }^{2}$ For the remainder of the paper, we report all of the conditional expectations under the normalization of $\mu_{\bar{\theta}}=0$. With $\mu_{\bar{\theta}} \neq 0$, the conditional expectations, such as (7)-(9) below, are given by a convex combination of the signal $s_{i}$ and prior mean $\mu_{\bar{\theta}}$. By normalizing $\mu_{\bar{\theta}}=0$, the statistical expressions become easier to read with minor loss of generality. By contrast, the description of the equilibrium in terms of mean and variance, as in Proposition 2, are always stated for $\mu_{\bar{\theta}} \in \mathbb{R}$.
} 


\section{Definition 1 (Bayes Nash Equilibrium).}

The strategy profile $a^{*}: \mathbb{R} \rightarrow \mathbb{R}$ forms a pure strategy symmetric Bayes Nash equilibrium if and only if:

$$
a^{*}\left(s_{i}\right)=\mathbb{E}\left[\theta_{i}+r \int a_{j}^{*}\left(s_{j}\right) d j \mid s_{i}\right], \forall s_{i} \in \mathbb{R} .
$$

The construction of a linear and symmetric equilibrium strategy in the multivariate normal environment is by now standard, see Vives [24] and Veldkamp [20]. Given an information structure $\lambda$, we denote the responsiveness, and in the linear strategy, the slope of the strategy in the signal $s_{i}$, by $w(\lambda)$.

\section{Proposition 1 (Noise Free BNE).}

For every noise free information structure $\lambda$, there is a unique Bayes Nash equilibrium and the strategy of each agent $i$ is linear in the signal $s_{i}$ :

$$
a_{i}^{*}\left(s_{i}\right)=w(\lambda) s_{i},
$$

with weight $w(\lambda)$ :

$$
w(\lambda)=\frac{\rho_{\theta \theta}(1-\lambda)+\left(1-\rho_{\theta \theta}\right) \lambda}{(1-r) \rho_{\theta \theta}(1-\lambda)^{2}+\lambda^{2}\left(1-\rho_{\theta \theta}\right)} .
$$

The responsiveness of the individual strategy is in general affected by the interaction parameter $r$, but in the special case of $r=0$, each agent solves an pure statistical prediction problem and the optimal weight corresponds to the Bayesian updating rule given by (9). If $r>0$, then the agents are in a game with strategic complements and respond stronger to the signal than Bayesian updating would suggest because of the inherent coordination motive with respect to the aggregate shock represented by the weight $\rho_{\theta \theta}(1-\lambda)^{2}$.

In every noise free information structure, the best response of each agent $i$ is always summarized by a scalar, $w(\lambda)$. To understand the impact the information structure has on the outcome of the equilibrium we provide a statistical description in terms of first and second moments of the variables (actions and payoff shocks) induced by an equilibrium. Given the information structure $\lambda$ and the linearity of the unique Bayes Nash equilibrium, we can immediately derive the properties of the joint distribution of the equilibrium variables.

\section{Proposition 2 (Moments of the Noise Free BNE).}

For every noise free information structure $\lambda$ :

1. the mean of the individual and the aggregate action is:

$$
\mathbb{E}\left[a_{i}\right] \triangleq \mu_{a}=\mu_{\theta} /(1-r)=\mu_{A} \triangleq \mathbb{E}[A]
$$


2. the variance of the individual action is:

$$
\operatorname{var}\left(a_{i}\right) \triangleq \sigma_{a}^{2}=w(\lambda)^{2}\left(\rho_{\theta \theta}(1-\lambda)^{2}+\left(1-\rho_{\theta \theta}\right) \lambda^{2}\right) \sigma_{\theta}^{2} ;
$$

3. and the variance of the aggregate action is:

$$
\operatorname{var}(A)=\operatorname{cov}\left(a_{i}, a_{j}\right) \triangleq \rho_{a a} \sigma_{a}^{2}=w(\lambda)^{2} \rho_{\theta \theta}(1-\lambda)^{2} \sigma_{\theta}^{2} .
$$

The mean of the individual and the aggregate action is only a function of the mean $\mu_{\theta}$ of the payoff shock $\theta_{i}$ and the interaction parameter $r$, and thus is invariant with respect to the composition of the payoff shocks in terms of idiosyncratic and aggregate shocks and invariant with respect to the information structure $\lambda$. By contrast, the second moments, respond to the composition of the shocks as represented by the correlation coefficient $\rho_{\theta \theta}$ and to the information structure $\lambda$. Given the normal distribution of the payoff shocks, and the linearity of the strategy, the variance and covariance terms are naturally the products of the weights, $\lambda$ and $w(\lambda)$, and the variance $\sigma_{\theta}^{2}$ of the fundamental uncertainty.

In the symmetric environment, the variance of the aggregate action, var $(A)$, coincides with covariance between any pair of actions by agents $i$ and $j, a_{i}$ and $a_{j}, \operatorname{cov}\left(a_{i}, a_{j}\right)$, and we denote the correlation coefficients by $\rho_{a a}$. The covariance between the action $a_{i}$ and the payoff shock $\theta_{i}$ of agent $i$, is given by:

$$
\operatorname{cov}\left(a_{i}, \theta_{i}\right) \triangleq \rho_{a \theta} \sigma_{a} \sigma_{\theta}=w(\lambda)\left(\rho_{\theta \theta}(1-\lambda)+\left(1-\rho_{\theta \theta}\right) \lambda\right) \sigma_{\theta}^{2}=\frac{\rho_{\theta \theta}(1-\lambda)+\left(1-\rho_{\theta \theta}\right) \lambda}{\sqrt{\rho_{\theta \theta}(1-\lambda)^{2}+\left(1-\rho_{\theta \theta}\right) \lambda^{2}}} \sigma_{\theta}^{2},
$$

whereas the covariance between the action $a_{i}$ of agent $i$ and the payoff shock $\theta_{j}$ of another agent $j$ is given by:

$$
\operatorname{cov}\left(a_{i}, \theta_{j}\right) \triangleq \rho_{a \phi} \sigma_{a} \sigma_{\theta}=w(\lambda)(1-\lambda) \rho_{\theta \theta} \sigma_{\theta}^{2}=\operatorname{cov}\left(a_{i}, \bar{\theta}\right)=\operatorname{cov}(A, \bar{\theta})
$$

We denote the corresponding correlation coefficients by $\rho_{a \theta}$ and $\rho_{a \phi}$, respectively.

As the above equalities indicate, in the symmetric environment, the covariance between the individual action $a_{i}$ of agent $i$ and the payoff shock $\theta_{j}$ of another agent $j, \operatorname{cov}\left(a_{i}, \theta_{j}\right)$, is equal to the covariance between the aggregate action $A$ and aggregate shock $\bar{\theta}$. After all, the idiosyncratic element vanishes in the covariance between $a_{i}$ and $\theta_{j}$, and what remains is the covariance between the common terms, $A$ and $\bar{\theta}$. Importantly though, the correlation coefficients $\rho_{a \phi}$ and $\rho_{A \bar{\theta}}$ differ as we can compute that

$$
\rho_{a \phi}=\frac{(1-\lambda) \rho_{\theta \theta}}{\sqrt{\left(\rho_{\theta \theta}(1-\lambda)^{2}+\left(1-\rho_{\theta \theta}\right) \lambda^{2}\right)}}
$$


but

$$
\rho_{A \bar{\theta}}=1
$$

Thus under any noise free information structure parametrized by $\lambda$, the aggregate action $A$ is perfectly correlated with the aggregate shock $\bar{\theta}$.

In their statistical content, the correlation coefficients reflect the direction in the relationship of any two random variables. Hence, the interaction parameter $r$ which only affects the slope of the equilibrium strategy, but not the composition of the signals, does not appear in the expression of the equilibrium correlation coefficients. Thus, for a given noise free information structure $\lambda$, we could, as an alternative to Proposition 2 completely characterize the Bayes Nash equilibrium in terms of the correlation coefficients $\left(\rho_{a a}, \rho_{a \theta}, \rho_{a \phi}\right)$. This triple only depends on the composition of the payoff shocks, $\rho_{\theta \theta}$ and the information structure $\lambda$. We could then recover all the moments of the equilibrium distribution, such as mean and variance, from the triple of correlation coefficients and the interaction parameter $r$.

\section{Individual Decisions and Aggregate Volatility}

We first consider aggregate volatility in the absence of any strategic interaction, and thus we are setting the strategic parameter $r$ equal to zero. While we focus on aggregate volatility, we will also report results for individual volatility and dispersion.

With $r=0$, the best response of each agent simply reflects a statistical prediction problem, namely to predict the payoff shock $\theta_{i}$ given the signal $s_{i}$ :

$$
a_{i}=\mathbb{E}\left[\theta_{i} \mid s_{i}\right]=\frac{(1-\lambda) \rho_{\theta \theta}+\lambda\left(1-\rho_{\theta \theta}\right)}{(1-\lambda)^{2} \rho_{\theta \theta}+\lambda^{2}\left(1-\rho_{\theta \theta}\right)} s_{i} .
$$

The individual prediction problem is more responsive to the signal $s_{i}$ if and only if the signal contains more information about the payoff shock $\theta_{i}$. As we observed, the noise free information structure $\lambda=1 / 2$ allows each agent to perfectly infer the individual payoff shock $\theta_{i}$. It follows that the responsiveness, and hence the variance of the individual action $\sigma_{a}^{2}$ is maximized at $\lambda=1 / 2$ :

$$
\sigma_{a}^{2}=\sigma_{\bar{\theta}}^{2}+\sigma_{\Delta \theta_{i}}^{2}
$$

Now, to the extent that the individual payoff shocks $\theta_{i}$ and $\theta_{j}$ are correlated, we find that even though each agent $i$ only solves an individual prediction problem, their actions are correlated by 
means of the underlying correlation of the individual payoff shocks. Under the information structure $\lambda=1 / 2$ the aggregate volatility is

$$
\sigma_{A}^{2}=\rho_{a a} \sigma_{a}^{2}=\sigma_{\bar{\theta}}^{2}
$$

We can now ask whether the aggregate volatility can reach higher levels under information structures different from $\lambda=1 / 2$. As the information structure departs from $\lambda=1 / 2$, we necessarily introduce a bias in the signal $s_{i}$ towards one of the two components of the payoff shock $\theta_{i}$. Clearly, the signal $s_{i}$ is losing informational quality with respect to the payoff shock $\theta_{i}$ as $\lambda$ moves away from $1 / 2$. Thus the individual prediction problem (16) is becoming noisier, and in consequence the response of the individual agent to the signal $s_{i}$ is attenuated. But a larger weight, $1-\lambda$, on the aggregate shock $\bar{\theta}$, may support correlation in the actions across agents, and thus support larger aggregate volatility. At the same time, the response of the agent is likely to be attenuated, and thus a trade-off appears between bias and loss of information. We then ask what is the maximal aggregate volatility that can be sustained across all noise free information structures.

\section{Proposition 3 (Maximal Aggregate Volatility).}

The maximal aggregate volatility:

$$
\max _{\lambda}\{\operatorname{var}(A)\}=\frac{\left(\sigma_{\bar{\theta}}+\sqrt{\sigma_{\bar{\theta}}^{2}+\sigma_{\Delta \theta_{i}}^{2}}\right)^{2}}{4},
$$

is achieved by the information structure $\lambda^{*}$ :

$$
\lambda^{*} \triangleq \underset{\lambda}{\arg \max }\{\operatorname{var}(A)\}=\frac{\sigma_{\bar{\theta}}}{2 \sigma_{\bar{\theta}}+\sqrt{\sigma_{\bar{\theta}}^{2}+\sigma_{\Delta \theta_{i}}^{2}}}<\frac{1}{2} .
$$

The aggregate volatility is maximized by an information structure which biases the signal towards the aggregate shock.

We recall from (2) that the total variance of the payoff shock $\theta_{i}$ is given by $\sigma_{\theta}^{2}=\sigma_{\bar{\theta}}^{2}+\sigma_{\Delta \theta_{i}}^{2}$. By a change of variable, we can express the variance of the idiosyncratic and aggregate components of the shocks in terms of the total variance of the payoff shock $\theta_{i}$ and the correlation coefficient $\rho_{\theta \theta}$, thus

$$
\sigma_{\bar{\theta}}^{2}=\rho_{\theta \theta} \sigma_{\theta}^{2}, \quad \sigma_{\Delta \theta_{i}}^{2}=\left(1-\rho_{\theta \theta}\right) \sigma_{\theta}^{2} .
$$

We can then express the information structure that maximizes the aggregate volatility in terms of the correlation coefficient $\rho_{\theta \theta}$ :

$$
\underset{\lambda}{\arg \max }\{\operatorname{var}(A)\}=\frac{\sqrt{\rho_{\theta \theta}}}{1+2 \sqrt{\rho_{\theta \theta}}},
$$


and the maximal volatility given by (17) can be expressed as:

$$
\max _{\lambda}\{\operatorname{var}(A)\}=\frac{1}{4}\left(1+\sqrt{\rho_{\theta \theta}}\right)^{2} \sigma_{\theta}^{2} .
$$

Thus, as we approach the environment with aggregate shocks only, $\rho_{\theta \theta} \rightarrow 1$ (and equivalently as the contribution from the idiosyncratic shock vanishes with $\sigma_{\Delta \theta_{i}}^{2} \rightarrow 0$ ), the maximal aggregate volatility of the actions coincides with the variance of the aggregate shock. This is achieved in a complete information equilibrium in which the action of each agent matches the realization of the payoff shock $\theta_{i}$. As all of the variance in the payoff shock $\theta_{i}$ stems from the aggregate shock, the variance of the individual action is attained by the variance of the average action.

More surprisingly, as we approach an environment with purely idiosyncratic shocks, the maximal aggregate volatility does not converge to zero, rather it is bounded away from 0 , and given by $\sigma_{\Delta \theta_{i}}^{2} / 4$, as stated in the following Corollary of Proposition 3.

Corollary 1 (Maximal Volatility with Aggregate or Idiosyncratic Shocks Only). The maximal volatility with aggregate shocks only is $\lim _{\sigma_{\Delta \theta_{i}}^{2} \rightarrow 0} \max _{\lambda}\{\operatorname{var}(A)\}=\sigma_{\bar{\theta}}^{2}$, with idiosyncratic shocks only is $\lim _{\sigma_{\bar{\theta}}^{2} \rightarrow 0} \max _{\lambda}\{\operatorname{var}(A)\}=\sigma_{\Delta \theta_{i}}^{2} / 4$.

If the individual payoff shock is increasingly dominated by the idiosyncratic shock, then the information structure puts more and more weight on the aggregate shock which itself has diminishing variance. The volatility maximizing information structure amplifies the response to the small aggregate shock and hence maintains a substantial correlation in the signals (and actions) across the agents, even though the payoff shocks are almost purely idiosyncratic and thus almost independent. If we consider the ratio of the weighted standard deviations of the aggregate and idiosyncratic shocks, where the weights are determined by the information structure $\lambda^{*}$, then we find that

$$
\frac{\left(1-\lambda^{*}\right) \sigma_{\bar{\theta}}}{\lambda^{*} \sigma_{\Delta \theta_{i}}}=\frac{1+\sqrt{\rho_{\theta \theta}}}{\sqrt{1-\rho_{\theta \theta}}},
$$

and in the limit as $\rho_{\theta \theta} \rightarrow 0$ (or equivalently as $\sigma_{\bar{\theta}} \rightarrow 0$ ),

$$
\lim _{\sigma_{\bar{\theta}} \rightarrow 0}\left\{\frac{\left(1-\lambda^{*}\right) \sigma_{\bar{\theta}}}{\lambda^{*} \sigma_{\Delta \theta_{i}}}\right\}=1 \text {. }
$$

Thus, the economy can maintain a large aggregate volatility even in the presence of vanishing aggregate payoff shocks by confounding the payoff relevant information about the idiosyncratic shock with the (in the limit) payoff irrelevant information about the aggregate shock.

In Section 3 we analyzed the equilibrium behavior in the noise free information structures assuming idiosyncratic and aggregate shocks, and hence confining the analysis to $\rho_{\theta \theta} \in(0,1)$. The above 
limit argument towards idiosyncratic shocks only suggests that as long as there is some arbitrarily small variation in the payoff shock, the signal can always amplify the informational importance of the shock much beyond its payoff importance. But, if there is no variance in either the idiosyncratic or the aggregate shock, then of course no amplification of those shocks is possible. Nonetheless, the limits in Corollary 1 can still be attained with zero variance in either one of the shocks, but now require noise in the signal that is payoff irrelevant. We illustrate this for the case of idiosyncratic payoff shocks only. Thus, consider an information structure in which each agent $i$ observes a signal that contains an error $\varepsilon$, common to all agents, with mean 0 and variance $\sigma_{\varepsilon}^{2}$,

$$
s_{i}=\Delta \theta_{i}+\varepsilon
$$

Given the signal $s_{i}$, the best response of agent $i$ is

$$
a_{i}=\mathbb{E}\left[\Delta \theta_{i} \mid s_{i}=\Delta \theta_{i}+\varepsilon\right]=\frac{\sigma_{\Delta \theta_{i}}^{2}}{\sigma_{\Delta \theta_{i}}^{2}+\sigma_{\varepsilon}^{2}} s_{i}
$$

and it follows that the realized average action $A$ is, as the idiosyncratic shock $\Delta \theta_{i}$ integrates out,

$$
A=\frac{\sigma_{\Delta \theta_{i}}^{2}}{\sigma_{\Delta \theta_{i}}^{2}+\sigma_{\varepsilon}^{2}} \varepsilon .
$$

The resulting variance of the average action, the aggregate volatility is:

$$
\sigma_{A}^{2}=\left(\frac{\sigma_{\Delta \theta_{i}}^{2}}{\sigma_{\Delta \theta_{i}}^{2}+\sigma_{\varepsilon}^{2}}\right)^{2} \sigma_{\varepsilon}^{2},
$$

and the aggregate volatility is maximized by setting the variance of the error term equal to the variance of the idiosyncratic shock:

$$
\sigma_{\varepsilon}^{2}=\sigma_{\Delta \theta_{i}}^{2} .
$$

This results in a positive level of aggregate volatility $\sigma_{A}^{2}$ driven by purely idiosyncratic payoff shocks $\sigma_{\Delta \theta_{i}}^{2}$ :

$$
\sigma_{A}^{2}=\frac{1}{4} \sigma_{\Delta \theta_{i}}^{2}
$$

The noisy information structure (21) thus achieves the limit of Corollary 1 with the noise to signal ratio of 1 implied by (23), which we derived earlier in (20) as the limiting ratio.

The maximal aggregate volatility is therefore achieved by an information structure that finds an optimal trade-off between biasing the information towards the aggregate shock, and here simply the common error, and maintaining responsiveness of agent $i$ towards the signal $s_{i}$ as given by the best 
response condition (22). Specifically, an increase in the variance $\sigma_{\varepsilon}^{2}$ of the error term leads to larger aggregate volatility only if the response of each agent to the signal does not become too attenuated. As the slope $\sigma_{\Delta \theta_{i}}^{2} /\left(\sigma_{\Delta \theta_{i}}^{2}+\sigma_{\varepsilon}^{2}\right)$ of the best response is decreasing in the variance $\sigma_{\varepsilon}^{2}$ of the error term, the idiosyncratic payoff shock can only absorb a finite variance of the error term, namely $\sigma_{\varepsilon}^{2}=\sigma_{\Delta \theta_{i}}^{2}$, before the response to the signal becomes too weak to generate additional aggregate volatility.

In the special cases of pure idiosyncratic or pure aggregate shocks, the payoff uncertainty is described completely by either $\Delta \theta_{i}$ or $\bar{\theta}$, and reduces from a two-dimensional to a one-dimensional space of uncertainty. In either case, across all $\lambda \in[0,1]$, there are only two possible noise free equilibrium outcomes. Namely, either players respond perfectly to the shock of the world (complete information) or players do not respond at all (zero information). For example, with idiosyncratic shocks only, that is $\rho_{\theta \theta}=0$, we have $\sigma_{\Delta \theta_{i}}^{2}=\sigma_{\theta}^{2}$, and $\sigma_{\bar{\theta}}^{2}=0$. Then, the signal $s_{i}$ is perfectly informative for all $\lambda \neq 0$ about the idiosyncratic shock, and we are effectively in a complete information setting. By contrast, if $\lambda=0$, then the signal $s_{i}$ is completely uninformative, and each agent makes a deterministic choice given the expected value $\mathbb{E}\left[\Delta \theta_{i}\right]=0$ of the shock. Correspondingly, for purely aggregate shocks, the critical value for which the information structure is completely uninformative is $\lambda=1$. Therefore there is a discontinuity at $\rho_{\theta \theta} \in\{0,1\}$ in the set of noise free Bayes Nash equilibria, but as the construction of the noisy information structure (21) suggests there is no discontinuity in the set of outcomes. The reason is simple and stems from the fact that as $\rho_{\theta \theta}$ approaches 0 or 1 , one of the dimensions of payoff uncertainty vanishes. Yet we should emphasize, that even as the payoff shocks approach the case of pure aggregate or pure idiosyncratic shocks, the part of the fundamental that becomes small can be arbitrarily amplified by the informational weight $\lambda$. For example, as $\rho_{\theta \theta} \rightarrow 1$, the idiosyncratic shock $\Delta \theta_{i}$ can still be amplified by letting $\lambda \rightarrow 1$ in the construction of the signal (6) above. Thus, the idiosyncratic shock $\Delta \theta_{i}$ acts like a purely idiosyncratic noise in an environment with aggregate shocks. After all, the idiosyncratic shock $\Delta \theta_{i}$ only affects the payoffs in a negligible way, but with a large enough weight, it has a non-negligible effect on the actions that the players take. This suggests that for the case in which the correlation of shocks approaches the case of pure aggregate or pure idiosyncratic shocks, there is no longer a sharp distinction between what is noise and what is fundamentals.

The dispersion of the individual action, $\Delta a_{i} \triangleq a_{i}-A$,is the volatility of the individual action beyond the aggregate volatility:

$$
\operatorname{var}\left(\Delta a_{i}\right)=\left(1-\rho_{a a}\right) \sigma_{a}^{2} .
$$


The analysis of the maximal dispersion is entirely symmetric to the one for aggregate volatility after we redefine the relevant variables in the obvious way:

$$
\tilde{\lambda} \triangleq(1-\lambda), \tilde{\rho}_{\theta \theta} \triangleq\left(1-\rho_{\theta \theta}\right)
$$

The result below then follows directly from Proposition 3.

Corollary 2 (Maximal Dispersion).

The maximal dispersion:

$$
\max _{\lambda}\left\{\operatorname{var}\left(\Delta a_{i}\right)\right\}=\frac{1}{4}\left(1+\sqrt{1-\rho_{\theta \theta}}\right)^{2} \sigma_{\theta}^{2}
$$

is achieved by the information structure $\lambda$ :

$$
\underset{\lambda}{\arg \max }\left\{\operatorname{var}\left(\Delta a_{i}\right)\right\}=\frac{1+\sqrt{1-\rho_{\theta \theta}}}{1+2 \sqrt{1-\rho_{\theta \theta}}}>\frac{1}{2} .
$$

\section{Strategic Decisions and Aggregate Volatility}

We proceed to analyze the aggregate volatility in the presence of strategic interaction. We thus return to the general model with $r \in(-\infty, 1)$ rather than $r=0$ as in the previous section. For each individual agent the responsiveness of the action to the signal will now depend on the informational content of the signal as well as the structure of the interaction.

An instructive benchmark for the responsiveness of the action to the signal is the behavior in the complete information equilibrium. Under complete information, each agent separately observes the idiosyncratic and aggregate shock, thus each player observes a noise free, but two-dimensional signal $\left(\bar{\theta}, \Delta \theta_{i}\right)$. The resulting equilibrium strategy is linear in the shocks and assigns weight 1 to the idiosyncratic shock $\Delta \theta_{i}$, and weight $1 /(1-r)$ to the aggregate shock $\bar{\theta}$, see (5). Given the linearity of the complete information strategy, a specific one-dimensional noise free information structure can replicate the outcome under the two-dimensional complete information structure. In particular, the one-dimensional information structure $\widehat{\lambda}$ with

$$
\widehat{\lambda} \triangleq \frac{1-r}{2-r}
$$

is the unique information structure $\lambda \in[0,1]$ such that for every realized pair of shocks, $\left(\Delta \theta_{i}, \bar{\theta}\right)$ and every signal realization $s_{i}$,

$$
s_{i}=\widehat{\lambda} \Delta \theta_{i}+(1-\lambda) \bar{\theta},
$$


the equilibrium action

$$
a_{i}^{*}\left(s_{i}\right)=\Delta \theta_{i}+\frac{\bar{\theta}}{1-r}
$$

coincides with the complete information equilibrium action. Thus for every level of strategic interaction, $r$, there is a noise free information structure $\widehat{\lambda}$ under which the agents' equilibrium behavior exactly mimics the complete information Nash equilibrium. Moreover, by inspecting the responsiveness to the signal, $w(\lambda)$, derived earlier in Proposition 1 , we find that $\hat{\lambda}$ is the unique information structure among all $\lambda \in[0,1]$ such that the responsiveness $w(\lambda)$ is invariant to the relative contribution of the aggregate and idiosyncratic shock, and hence invariant with respect to the value of $\rho_{\theta \theta}$, namely

$$
w(\widehat{\lambda})=\frac{1}{\widehat{\lambda}}=\frac{2-r}{1-r} .
$$

In particular, the Bayes Nash equilibrium under the information structure $\widehat{\lambda}$ always reproduces the complete information outcome, independent of the composition of the shocks.

We now decompose the responsiveness of the individual action $a_{i}$ into the components of the payoff shock $\theta_{i}$, namely the idiosyncratic shock $\Delta \theta_{i}$ and the aggregate shock $\bar{\theta}$. Given the multivariate normal distribution, the responsiveness of the action can be expressed in terms of the covariance:

$$
\frac{\partial \mathbb{E}\left[a_{i} \mid \Delta \theta_{i}\right]}{\partial \Delta \theta_{i}}=\frac{\operatorname{cov}\left(a_{i}, \Delta \theta_{i}\right)}{\sigma_{\Delta \theta}^{2}}=\lambda w(\lambda), \quad \frac{\partial \mathbb{E}\left[a_{i} \mid \bar{\theta}\right]}{\partial \bar{\theta}}=\frac{\operatorname{cov}\left(a_{i}, \bar{\theta}\right)}{\sigma_{\bar{\theta}}^{2}}=(1-\lambda) w(\lambda) .
$$

Proposition 4 (Responsiveness to Fundamentals).

In the noise free Bayes Nash equilibrium with information structure $\lambda$ :

1. $\lambda \in(\widehat{\lambda}, 1) \Leftrightarrow \frac{\operatorname{cov}\left(a_{i}, \Delta \theta_{i}\right)}{\sigma_{\Delta \theta}^{2}}>1$;

2. $\lambda \in(0, \widehat{\lambda}) \Leftrightarrow \frac{\operatorname{cov}\left(a_{i}, \bar{\theta}\right)}{\sigma_{\bar{\theta}}^{2}}>\frac{1}{1-r}$.

Thus, the responsiveness of the action to each component of the payoff shock is determined by the weight $\lambda$ that the signal assigns relative to complete information benchmark $\hat{\lambda}$. For any given information structure $\lambda$, the responsiveness is stronger than in the complete information environment for exactly one of the components, and weaker for the other.

We recall from the analysis in the previous section that with purely aggregate shocks, any residual uncertainty about the payoff shock inevitably reduced the responsiveness of the individual agent to the aggregate shock, and ultimately reduced the aggregate responsiveness. Similarly, with idiosyncratic shocks only, the residual uncertainty attenuated the responsiveness to his payoff 
shock $\theta_{i}$. By contrast, in the joint presence of idiosyncratic and aggregate shocks, the interaction between the idiosyncratic and the aggregate shock can correlate the responsiveness of the agents without attenuating the individual response to one of the components, thus leading to a greater responsiveness to one component of the shock than could be achieved under complete information. Intuitively, this stems from the fact that part of the responsiveness to one of the components is loaded onto the other component.

In Figure 1 we plot the responsiveness for the case of $\rho_{\theta \theta}=0.5$ and different interaction parameters $r$. The threshold values $\widehat{\lambda}_{r}$ simply corresponds to the critical value $\widehat{\lambda}$ for each of the considered interaction parameters $r \in\left\{-\frac{3}{4}, 0,+\frac{3}{4}\right\}$. The horizontal black lines represent the responsiveness to the aggregate shock $\bar{\theta}$ in the complete information equilibrium which is equal to $1 /(1-r)$, and the responsiveness to the idiosyncratic part, which is always equal to 1. By contrast, the red curves represent the responsiveness to the aggregate shock along the noise free equilibrium, and the blue curves represent the responsiveness to the idiosyncratic shock. Thus if $\lambda<\widehat{\lambda}$, then the responsive-

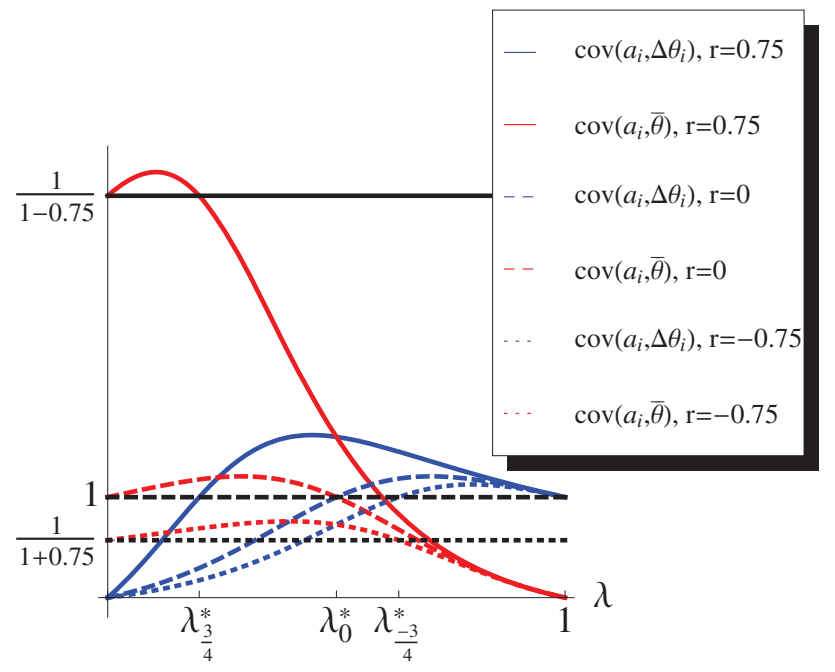

Figure 1: Responsiveness to Fundamentals for $\rho_{\theta \theta}=1 / 2$

ness to the aggregate shock $\bar{\theta}$ is larger than in the complete information equilibrium, and conversely for $\Delta \theta_{i}$. Moreover, we observe that the maximum responsiveness to the aggregate shock is never attained under either the complete information equilibrium or at the boundary values of $\lambda$, at 0 or 1 . This immediately implies that the responsiveness is not monotonic in the informational content. We now provide some comparative static results with respect to the strategic environment represented 
by $r$.

\section{Proposition 5 (Informational Weight and Maximal Volatility).}

For all $\rho_{\theta \theta} \in(0,1)$ :

1. the informational weights $\lambda$ that maximize the second moments satisfy:

$$
\operatorname{argmax}_{\lambda}\left\{\left(1-\rho_{a a}\right) \sigma_{a}^{2}\right\}>\operatorname{argmax}_{\lambda}\left\{\sigma_{a}^{2}\right\}>\operatorname{argmax}_{\lambda}\left\{\rho_{a a} \sigma_{a}^{2}\right\} ;
$$

2. the informational weights $\lambda$ that maximize the second moments: $\operatorname{argmax}_{\lambda} \sigma_{a}^{2}, \operatorname{argmax}_{\lambda} \rho_{a a} \sigma_{a}^{2}$, $\operatorname{argmax}_{\lambda}\left(1-\rho_{a a}\right) \sigma_{a}^{2}$ are strictly decreasing in $r$;

3. the maximal second moments: $\max _{\lambda} \sigma_{a}^{2}, \max _{\lambda} \rho_{a a} \sigma_{a}^{2}, \max _{\lambda}\left(1-\rho_{a a}\right) \sigma_{a}^{2}$ are strictly increasing in $r$.

Thus, the maximal volatility, both individual and aggregate, is increasing in the level of complementarity $r$. Even the maximal dispersion is increasing in $r$. In the equilibrium with maximum dispersion, the agents confound the idiosyncratic and aggregate component of the payoff shock and overreact to the idiosyncratic part, this effect increases with $r$. This implies that the responsiveness to the aggregate shock $\bar{\theta}$ increases, and hence the overreaction to the idiosyncratic shock $\Delta \theta_{i}$ increases as well. Moreover, the optimal weight on the aggregate shock increases in $r$ for all of the second moments.

\section{Proposition 6 (Aggregate Volatility).}

The maximal aggregate volatility is given by:

$$
\max _{\lambda}\left\{\rho_{a a} \sigma_{a}^{2}\right\}=\frac{\sigma_{\Delta \theta_{i}}^{4}}{4\left(\sqrt{\sigma_{\bar{\theta}}^{2}+(1-r) \sigma_{\Delta \theta_{i}}^{2}}-\sigma_{\bar{\theta}}\right)^{2}}
$$

and is strictly increasing and without bound in the idiosyncratic uncertainty $\sigma_{\Delta \theta_{i}}^{2}$.

Importantly, the maximum aggregate volatility is not bounded anymore by the aggregate volatility of the complete information equilibrium. In fact, the aggregate volatility is increasing without bounds in the variance of the idiosyncratic shock even in the absence of variance of the aggregate shock $\bar{\theta}$. These results are in stark contrast to the complete information equilibrium in which the aggregate volatility is unaffected by the variance of the idiosyncratic shock. It illustrates in a simple way that the aggregate volatility may stem from uncertainty about either the aggregate or the idiosyncratic fundamental. As in the case of individual decision-making, we can consider the limits of the aggregate volatility as we approach a model of aggregate or idiosyncratic shocks only.

Corollary 3 (Maximal Volatility with Aggregate or Idiosyncratic Shocks Only). The maximal volatility with only aggregate shocks is $\lim _{\sigma_{\Delta \theta_{i}}^{2} \rightarrow 0} \max _{\lambda}\{\operatorname{var}(A)\}=\sigma_{\bar{\theta}}^{2} /(1-r)^{2}$ and with only idiosyncratic shocks is $\lim _{\sigma_{\bar{\theta}}^{2} \rightarrow 0} \max _{\lambda}\{\operatorname{var}(A)\}=\sigma_{\Delta \theta_{i}}^{2} /(4(1-r))$. 
Confounding Information. The idea that confounding shocks can lead to overreaction goes back at least to Lucas [14]. In a seminal contribution, he shows how monetary shocks can have a real effect in the economy, even when under complete information monetary shocks would have no real effect. As agents observe just a one-dimensional signal (a price) that confounds two shocks, namely the labor market shock and the monetary supply shock, they necessarily respond to the two shocks in the same way. By contrast, under complete information they would condition their hiring decisions only on the labor market conditions. Yet the one-dimensional signal does not allow them to disentangle both shocks and in equilibrium they respond to both shocks. Thus, this can be seen as an overreaction to monetary shocks due to "informational frictions". The idea has been present also in more recent papers. For example, Venkateswaran [21] uses a similar idea to show how firms can have an excess reaction to aggregate shocks when these are confounded with idiosyncratic shocks or Mackowiak and Wiederholt [15] who derive informational frictions in a model of rational inattention.

In a recent contribution, Angeletos and La'O [4] consider an economy with purely idiosyncratic payoff shocks that still displays aggregate fluctuations. Each agent is assumed to know the realization of his individual payoff shock but only interacts with a specific trading partner rather than the aggregate market. Now, even though the payoff uncertainty is purely idiosyncratic, and each agent knows his own payoff shock, the pairwise interaction leaves each agent uncertain about the action of his trading partner. It is this uncertainty that can be affected by a common noise term, and hence generate aggregate volatility across the agents. They interpret this common noise term as sentiments which generate aggregate fluctuations.

The current analysis allow us to extract some very simple intuitions on when informational frictions can have a big effect on aggregate outcomes. We saw that as we approach a world of idiosyncratic uncertainty $\left(\rho_{\theta \theta} \rightarrow 0\right)$, the maximum aggregate volatility is bounded away from 0 and it is achieved by a noise free equilibria. The information structure amplifies the aggregate shock that has small variance and leads to a big response by the agents provided that the informational weight on the aggregate shock is sufficiently large. Thus, we find that in a model with idiosyncratic uncertainty such as Angeletos and La'O [4], any arbitrarily small aggregate shock can have a huge effect, that it can be amplified if it receives a sufficiently large weight in the signal of the agents. 


\section{Equilibrium Behavior for All Information Structures}

Until now we have analyzed the outcomes under the Bayes Nash equilibrium for a very special class of information structures. By contrast, we now describe the equilibrium behavior for all (symmetric and normally distributed) information structures. Thus we now allow for noisy rather than noise free information structures, and we allow for multidimensional rather than one-dimensional signals. Moreover, the signals, both across dimensions and across agents can have arbitrary correlation structures.

\subsection{Definition of Bayes Correlated Equilibrium}

In order to describe the equilibrium behavior across all possible information structures, we introduce a solution concept, Bayes correlated equilibrium, that describes the behavior (and outcomes) independent of the specific information structure that the agents may have access to. The set of Bayes correlated equilibria has the advantage that it can be completely described by a small set of inequalities on the first and second moments of the equilibrium distribution.

Definition 2 (Bayes Correlated Equilibrium).

The variables $\left(\theta_{i}, \bar{\theta}, a_{i}, A\right)$ form a symmetric and normally distributed Bayes correlated equilibrium if their joint distribution is given by a multivariate normal distribution and for all $i$ and $a_{i}$ :

$$
a_{i}=r \mathbb{E}\left[A \mid a_{i}\right]+\mathbb{E}\left[\theta_{i} \mid a_{i}\right]
$$

The Bayes correlated equilibrium requires that the joint distribution of shocks and actions is such that for every action $a_{i}$ in the support of the joint distribution the best response satisfies condition (27). We emphasize that the equilibrium notion does not refer to any information structure or signals. It is intentionally defined without reference to any specific information structure in contrast to the notion of Bayes Nash equilibrium that is always defined with reference to a specific information structure. The only informational restrictions that are imposed by the above equilibrium notion are that: $(i)$ the marginal distribution over the payoff shocks $\left(\theta_{i}, \bar{\theta}\right)$ coincides with common prior, and $(i i)$ each agent conditions on the information contained in joint distribution, and hence when choosing action $a_{i}$ forms the conditional expectation. The notion of Bayes correlated equilibrium was defined earlier in Bergemann and Morris [8], [9]. In the present context, we use it to obtain bounds on the volatility of equilibrium without reference to a specific information structure to begin with. 


\subsection{Characterization of Bayes Correlated Equilibria}

We begin the analysis of the Bayes correlated equilibrium by reducing the dimensionality of the variance-covariance matrix. We appeal to the symmetry condition to express the aggregate variance in terms of the individual variance and the correlation between individual terms. Just as we described the variance $\sigma_{\bar{\theta}}^{2}$ of the aggregate shock $\bar{\theta}$ in terms of the covariance between any two individual payoff shocks, or $\sigma_{\bar{\theta}}^{2}=\rho_{\theta \theta} \sigma_{\theta}^{2}$, we can describe the variance of aggregate action $\sigma_{A}^{2}$ in terms of the covariance of any two individual actions, or $\sigma_{A}^{2}=\rho_{a a} \sigma_{a}^{2}$. In the discussion following Proposition 2, we introduced the correlation coefficient between action $a_{i}$ and payoff shock $\theta_{i}$ of player $i$ by $\rho_{a \theta}$ :

$$
\operatorname{cov}\left(a_{i}, \theta_{i}\right) \triangleq \rho_{a \theta} \sigma_{a} \sigma_{\theta}
$$

and the correlation coefficient between the action $a_{i}$ of agent $i$ and the payoff shock $\theta_{j}$ of a different agent $j$ by $\rho_{a \phi}$ :

$$
\operatorname{cov}\left(a_{i}, \theta_{j}\right) \triangleq \rho_{a \phi} \sigma_{a} \sigma_{\theta}
$$

These three correlation coefficients, $\left(\rho_{a a}, \rho_{a \theta}, \rho_{a \phi}\right)$, parameterize the entire variance-covariance matrix, denoted by $\mathbb{V}$, of the joint distribution of $\left(\theta_{i}, \bar{\theta}, a_{i}, A\right)$. Now, the covariance between a purely idiosyncratic random variable and an aggregate random variable is always 0 . This implies that both the covariance between the aggregate action $A$ and the payoff shock $\theta_{j}$ of player $j$ and the covariance between the agent $i$ 's action, $a_{i}$, and the aggregate shock to the payoff shock, $\bar{\theta}$, are the same as the covariance between the action of player $i$ and the payoff shock $\theta_{j}$ of player $j$, or $\rho_{a \phi} \sigma_{a} \sigma_{\theta}$. Thus we can reduce the number of variance terms, and in particular the number of correlation coefficients needed to describe the variance-covariance matrix $\mathbb{V}$ without any loss of generality.

\section{Lemma 1 (Symmetric Bayes Correlated Equilibrium).}

The variables $\left(\theta_{i}, \bar{\theta}, a_{i}, A\right)$ form a symmetric and normally distributed Bayes correlated equilibrium if and only if there exist parameters of the first and second moments, $\left(\mu_{a}, \sigma_{a}, \rho_{a a}, \rho_{a \theta}, \rho_{a \phi}\right)$, such that the joint distribution is given by:

$$
\left(\begin{array}{c}
\theta_{i} \\
\bar{\theta} \\
a_{i} \\
A
\end{array}\right) \sim \mathcal{N}\left(\left(\begin{array}{l}
\mu_{\theta} \\
\mu_{\theta} \\
\mu_{a} \\
\mu_{a}
\end{array}\right),\left(\begin{array}{cccc}
\sigma_{\theta}^{2} & \rho_{\theta \theta} \sigma_{\theta}^{2} & \rho_{a \theta} \sigma_{a} \sigma_{\theta} & \rho_{a \phi} \sigma_{a} \sigma_{\theta} \\
\rho_{\theta \theta} \sigma_{\theta}^{2} & \rho_{\theta \theta} \sigma_{\theta}^{2} & \rho_{a \phi} \sigma_{a} \sigma_{\theta} & \rho_{a \phi} \sigma_{a} \sigma_{\theta} \\
\rho_{a \theta} \sigma_{a} \sigma_{\theta} & \rho_{a \phi} \sigma_{a} \sigma_{\theta} & \sigma_{a}^{2} & \rho_{a a} \sigma_{a}^{2} \\
\rho_{a \phi} \sigma_{a} \sigma_{\theta} & \rho_{a \phi} \sigma_{a} \sigma_{\theta} & \rho_{a a} \sigma_{a}^{2} & \rho_{a a} \sigma_{a}^{2}
\end{array}\right)\right)
$$

and for all $i$ and $a_{i}$ :

$$
a_{i}=r \mathbb{E}\left[A \mid a_{i}\right]+\mathbb{E}\left[\theta_{i} \mid a_{i}\right]
$$


We can now find restrictions on the first and second moments of $a_{i}$ using the best response condition (29). By taking expectations of (29) and using the law of iterated expectations, we get:

$$
\mu_{a}=r \mu_{a}+\mu_{\theta} \Leftrightarrow \mu_{a}=\frac{\mu_{\theta}}{1-r}
$$

If we multiply (29) by $a_{i}$ and take expectations we get:

$$
\mathbb{E}\left[a_{i}^{2}\right]=r \mathbb{E}\left[a_{i} A\right]+\mathbb{E}\left[a_{i} \theta_{i}\right]
$$

Using (30) we obtain a similar condition for the variance of $a_{i}$ :

$$
\sigma_{a}^{2}=\operatorname{rov}\left(a_{i}, A\right)+\operatorname{cov}\left(a_{i}, \theta_{i}\right) \Leftrightarrow \sigma_{a}=\frac{\rho_{a \theta} \sigma_{\theta}}{1-r \rho_{a a}} .
$$

We thus have a complete determination of the individual mean and variance. Note that these conditions do not depend at all on the joint normality of $\left(a_{i}, A, \theta_{i}, \bar{\theta}\right)$ as they only used the law of iterated expectations. Moreover, it is easy to see how one could get similar additional restrictions for the higher moments of $a_{i}$ which would be relevant for the non-normal case. Now, for $\mathbb{V}$ to be a valid variance-covariance matrix, it has to be positive semidefinite, and this imposes restrictions on the remaining covariance terms.

\section{Proposition 7 (Characterization).}

A multivariate normal distribution of $\left(\theta_{i}, \bar{\theta}, a_{i}, A\right)$ is a symmetric Bayes correlated equilibrium if and only if :

1. the mean of the individual action is:

$$
\mu_{a}=\mu_{\theta} /(1-r)
$$

2. the standard deviation of the individual action is:

$$
\sigma_{a}=\frac{\rho_{a \theta} \sigma_{\theta}}{1-r \rho_{a a}} \geq 0
$$

3. the correlation coefficients $\rho_{a a}, \rho_{a \theta}, \rho_{a \phi}$ satisfy $\rho_{a a}, \rho_{a \theta} \geq 0$ and the inequalities:

$$
\text { (i) }\left(\rho_{a \phi}\right)^{2} \leq \rho_{\theta \theta} \rho_{a a}, \quad(i i) \quad\left(1-\rho_{a a}\right)\left(1-\rho_{\theta \theta}\right) \geq\left(\rho_{a \theta}-\rho_{a \phi}\right)^{2} .
$$

We thus identified necessary and sufficient conditions for the random variables $\left(\theta_{i}, \bar{\theta}, a_{i}, A\right)$ to form a Bayes correlated equilibrium. These conditions can be separated into two distinct sets of requirements: the first set of conditions follow directly from the best response condition (27) and merely rely on the linearity of the best response; the second set of conditions are purely statistical 
conditions that require that the variance-covariance matrix $\mathbb{V}$ of the joint multivariate distribution constitutes a valid variance-covariance matrix, namely that it is positive semidefinite.

Importantly, both sets of conditions are necessary independent of the assumption of normally distributed payoff uncertainty. The normality assumption simply ensures that the equilibrium distributions are completely determined by the first and second moments. Thus, the normality assumptions allows us to describe the set of Bayes correlated equilibria in terms of restrictions that are necessary and sufficient. Nevertheless, for any arbitrary distribution of payoff shocks and equilibrium actions, the aggregate volatility provided in Proposition 6 will remain a valid bound on aggregate volatility.

There are two aspects of Proposition 7 that we should highlight. First, the mean $\mu_{a}$ of the individual action (and a fortiori the mean of the aggregate action $A$ ) is completely pinned down by the aggregate payoff shock. This implies that any differences across Bayes correlated equilibria must manifest themselves in the second moments only. Second, the restrictions on the equilibrium correlation coefficients do not at all depend on the interaction parameter $r$. The restrictions on the set of equilibrium correlations are purely statistical and stem from the condition that the variancecovariance matrix $\mathbb{V}$ forms a positive semidefinite matrix. By contrast, the mean $\mu_{a}$ and the variance $\sigma_{a}^{2}$ of the individual actions do depend on the interaction parameter $r$, as they are determined by the best response condition (29).

We will show in Section 7 that the disentanglement of the set of feasible correlations and the interaction parameter is possible only if we allow for all possible information structures, i.e. when we do not impose any restrictions on the private information that agents may have.

In the special case of pure idiosyncratic or pure aggregate shocks the set of outcomes in terms of the correlation coefficients $\left(\rho_{a a}, \rho_{a \theta}, \rho_{a \phi}\right)$ reduces to a two-dimensional set. The reduction in dimensionality arises as the correlation coefficient $\rho_{a \phi}$ of action $a_{i}$ and the payoff shock $\theta_{j}$ is either zero (in the absence of aggregate shocks) or equal $\rho_{a \theta}$ (in the absence of idiosyncratic shocks), and thus redundant in either case. In the case of pure aggregate shocks, $\rho_{\theta \theta}=1$, the conditions in (34) reduce to $\rho_{a \phi}=\rho_{a \theta}$, and $\rho_{a \theta}^{2} \leq \rho_{a a}$ as established earlier in Bergemann and Morris [8].

\subsection{Equivalence between Bayes Nash and Bayes Correlated Equilibrium}

Next we describe the relationship between the joint distributions of $\left(\theta_{i}, \bar{\theta}, a_{i}, A\right)$ that can arise as Bayes correlated equilibria and the distributions that can arise as Bayes Nash equilibria for some information structure $\mathcal{I}=\left\{S_{i}\right\}_{i \in[0,1]}$. In contrast to the restriction to one-dimensional noise free information structure made in Section 3, we now allow for a much larger class of information 
structures, including noisy and multidimensional information structures. In fact, for the present purpose, it is sufficient to merely require that the associated symmetric equilibrium strategy $\left\{a_{i}\right\}_{i \in[0,1]}$ : $a_{i}: S_{i} \rightarrow \mathbb{R}$ forms a multivariate normal distribution.

\section{Proposition 8 (Equivalence).}

The variables $\left(\theta_{i}, \bar{\theta}, a_{i}, A\right)$ form a (normal) Bayes correlated equilibrium if and only if there exists some information structure $\mathcal{I}$ under which the variables $\left(\theta_{i}, \bar{\theta}, a_{i}, A\right)$ form a Bayes Nash equilibrium.

The important insight of the equivalence is that the set of outcomes that can be achieved as a Bayes Nash equilibrium for some information structure can equivalently be described as a Bayes correlated equilibrium. Thus, the solution concept of Bayes correlated equilibrium allows us to study the set of outcomes that can be achieved as a Bayes Nash equilibrium, importantly without the need to specify a specific information structure. In Bergemann and Morris [9], we establish the equivalence between Bayes correlated equilibrium and Bayes Nash equilibrium for canonical finite games and arbitrary information structures (see Theorem 1 there). The above proposition specializes the proof to an environment with linear best responses and symmetrically normally distributed payoff shocks and actions.

We will discuss specific information structures and their associated equilibrium behavior in Section 7. Here, we describe a one-dimensional class of signals that is already sufficiently rich to "decentralize" the entire set of Bayes correlated equilibria as Bayes Nash equilibria. For this, we enlarge the set of noise free structures studied in Section 3 by allowing the weights on $\Delta \theta_{i}$ and $\bar{\theta}$ to have different signs, and adding noise:

$$
s_{i}=\lambda \Delta \theta_{i}+(1-|\lambda|) \bar{\theta}+\varepsilon_{i},
$$

where $\lambda \in[-1,1]$ and $\varepsilon_{i}$ is normally distributed with mean zero and variance $\sigma_{\varepsilon}^{2}{ }^{3}$. Similarly to the definition of the payoff shocks, the individual error term $\varepsilon_{i}$ can have a common and an idiosyncratic component, respectively:

$$
\bar{\varepsilon} \triangleq \mathbb{E}_{i}\left[\varepsilon_{i}\right], \quad \Delta \varepsilon_{i} \triangleq \varepsilon_{i}-\bar{\varepsilon} .
$$

\footnotetext{
${ }^{3}$ The weight $\lambda$ on the idiosyncratic shock is now allowed to take on negative values, and thus the weights on the idiosyncratic and the aggregate shock can have different signs. By expanding the class of information structures relative to those considered in Section 3, we expand the set of feasible information structures. This permits for equilibrium correlation structures in which either $\rho_{a \theta}<\rho_{\alpha \phi}$ or $\rho_{a \phi}<0$.
} 
Thus, the joint distribution of the shocks and signals is given by:

$$
\left(\begin{array}{c}
\Delta \theta_{i} \\
\bar{\theta} \\
\Delta \varepsilon_{i} \\
\bar{\varepsilon}
\end{array}\right) \sim \mathcal{N}\left(\left(\begin{array}{c}
0 \\
\mu_{\theta} \\
0 \\
0
\end{array}\right),\left(\begin{array}{cccc}
\left(1-\rho_{\theta \theta}\right) \sigma_{\theta}^{2} & 0 & 0 & 0 \\
0 & \rho_{\theta \theta} \sigma_{\theta}^{2} & 0 & 0 \\
0 & 0 & \left(1-\rho_{\varepsilon \varepsilon}\right) \sigma_{\varepsilon}^{2} & 0 \\
0 & 0 & 0 & \rho_{\varepsilon \varepsilon} \sigma_{\varepsilon}^{2}
\end{array}\right)\right)
$$

and the standard deviation $\sigma_{\varepsilon}>0$ and the correlation coefficient $\rho_{\varepsilon \varepsilon} \in[0,1]$ are the parameters of the fully specified information structure $\mathcal{I}=\left\{\mathcal{I}_{i}\right\}_{i \in[0,1]}$, together with the confounding parameter $\lambda$. We observe that the dimensionality of information structure $\mathcal{I}$, given by (35) and (36), and thus parametrized by $\left(\lambda, \sigma_{\varepsilon}, \rho_{\varepsilon \varepsilon}\right)$, matches the dimensionality of the Bayes correlated equilibrium expressed in terms of the correlation coefficients $\left(\rho_{a a}, \rho_{a \theta}, \rho_{a \phi}\right)$.

Proposition 9 (Informational Decentralization).

The variables $\left(\theta_{i}, \bar{\theta}, a_{i}, A\right)$ form a (normal) Bayes correlated equilibrium if and only if there exist some information structure $\left(\lambda, \sigma_{\varepsilon}^{2}, \rho_{\varepsilon \varepsilon}\right)$ under which the variables $\left(\theta_{i}, \bar{\theta}, a_{i}, A\right)$ form a Bayes Nash equilibrium.

The class of one-dimensional signals described by (35) is thus rich enough to "informationally decentralize" in the sense that every Bayes correlated equilibrium distribution can be reproduced as a Bayes Nash equilibrium distribution for some choice of the parameters $\left(\lambda, \sigma_{\varepsilon}, \rho_{\varepsilon \varepsilon}\right)$ that describe the family of information structures given by (35). This illustrates how the equilibrium conditions of the Bayes correlated equilibrium, in particular the conditioning in the best response (27) encode in the joint distribution information that is made explicit by the information structure of the corresponding Bayes Nash equilibrium.

\subsection{The Boundary of Bayes Correlated Equilibria}

We characterized the entire set of Bayes correlated equilibria in Proposition 7. The mean and the variance of the individual action were determined by the equalities (32) and (33) and the correlation coefficients $\left(\rho_{a a}, \rho_{a \theta}, \rho_{a \phi}\right)$ were restricted by the two inequalities given by (34). We now provide a characterization of the boundary of the set of Bayes correlated equilibria in terms of the two inequalities which only involve the correlation coefficients $\left(\rho_{a a}, \rho_{a \theta}, \rho_{a \phi}\right)$.

Definition 3 (Boundary of Bayes Correlated Equilibrium).

The correlation coefficients $\left(\rho_{a a}, \rho_{a \theta}, \rho_{a \phi}\right)$ are on the (upper) boundary of the Bayes correlated equilibrium set if they satisfy (34) and there is no other triple of coefficients $\left(\rho_{a a}, \rho_{a \theta}^{\prime}, \rho_{a \phi}^{\prime}\right)$, also satisfying (34), such that:

$$
\left(\rho_{a \theta}^{\prime}, \rho_{a \phi}^{\prime}\right) \geq\left(\rho_{a \theta}, \rho_{a \phi}\right), \text { and }\left(\rho_{a \theta}^{\prime}, \rho_{a \phi}^{\prime}\right) \neq\left(\rho_{a \theta}, \rho_{a \phi}\right)
$$


We refer to any triple $\left(\rho_{a a}, \rho_{a \theta}, \rho_{a \phi}\right)$ that satisfies the conditions of Definition 3 as an element of the upper boundary. For any attainable correlation between actions, $\rho_{a a}$, there is no other Bayes correlated equilibrium $\left(\rho_{a a}, \rho_{a \theta}^{\prime}, \rho_{a \phi}^{\prime}\right)$ that achieves a weakly higher correlation with the idiosyncratic shock $\Delta \theta_{i}$ and the aggregate shock $\bar{\theta}$, and strictly higher for at least one of the two shocks.

Now, necessary conditions for a triple $\left(\rho_{a a}, \rho_{a \theta}, \rho_{a \phi}\right)$ to be on the boundary of the Bayes correlated equilibrium set is that both inequalities (34) are satisfied as equalities, and the restriction to the positive root of the quadratic equations yields the sufficient condition.

\section{Proposition 10 (Boundary of Bayes Correlated Equilibria).}

The correlation coefficients $\left(\rho_{a a}, \rho_{a \theta}, \rho_{a \phi}\right)$ form the boundary of the Bayes correlated equilibrium if and only if

$$
\left\{\left(\rho_{a a}, \rho_{a \theta}, \rho_{a \phi}\right) \in[0,1]^{3}: \rho_{a \theta}=\sqrt{\rho_{a a} \rho_{\theta \theta}}+\sqrt{\left(1-\rho_{\theta \theta}\right)\left(1-\rho_{a a}\right)}, \rho_{a \phi}=\sqrt{\rho_{a a} \rho_{\theta \theta}}\right\} .
$$

The boundary of the Bayes correlated equilibria is described by equation (37) which identifies $\rho_{a \theta}$ and $\rho_{a \phi}$ as a function of $\rho_{a a}$ for a given composition of idiosyncratic and aggregate shocks, $\rho_{\theta \theta}$. Accordingly, the correlation coefficient $\rho_{a \theta}$ between action $a_{i}$ and shock $\theta_{i}$ of agent $i$ is the sum of two roots. The roots arise from the aggregate and the idiosyncratic shock in the agents' actions and the agents' shocks, $\rho_{a a} \rho_{\theta \theta}$ and $\left(1-\rho_{a a}\right)\left(1-\rho_{\theta \theta}\right)$, respectively. The set of attainable correlations is restricted by the composition of the payoff shocks as (37) depends explicitly on $\rho_{\theta \theta}$. Figure 2 visualizes the boundary of the equilibrium set in the two-dimensional space of correlation coefficients $\left(\rho_{a a}, \rho_{a \theta}\right)$ for different compositions of the shocks, $\rho_{\theta \theta}$. The left panel represents the case of $\rho_{\theta \theta}=1 / 2$, whereas the right panel represents $\rho_{\theta \theta}=1 / 4$ and $\rho_{\theta \theta}=3 / 4$. The right panel illustrates how the composition of the payoff shocks, $\rho_{\theta \theta}$, impacts the relationship between $\rho_{a a}$ and $\rho_{a \theta}$ relative to the symmetric condition with $\rho_{\theta \theta}=1 / 2$.

We can decompose the action of each agent in terms of his responsiveness to the idiosyncratic shock $\Delta \theta_{i}$ and the aggregate shock $\bar{\theta}$, and any residual responsiveness has to be attributed to noise, see (25). The action $a_{i}$ itself also has an idiosyncratic and an aggregate component as $a_{i}=A+\Delta a_{i}$. The conditional variance of these components of $a_{i}$ can be expressed in terms of the correlation coefficients $\left(\rho_{a a}, \rho_{a \theta}, \rho_{a \phi}, \rho_{\theta \theta}\right)$, which are subject to the restrictions of Proposition 7. By using the familiar property of the multivariate normal distribution for the conditional variance, we obtain a diagonal matrix:

$$
\operatorname{var}\left[\begin{array}{c|c}
\Delta a_{i} & \Delta \theta_{i} \\
A & \bar{\theta}
\end{array}\right]=\sigma_{a}^{2}\left(\begin{array}{cc}
\left(1-\rho_{a a}\right)-\frac{\left(\rho_{a \theta}-\rho_{a \phi}\right)^{2}}{1-\rho_{\theta \theta}} & 0 \\
0 & \rho_{a a}-\frac{\rho_{a \phi}^{2}}{\rho_{\theta \theta}}
\end{array}\right) .
$$



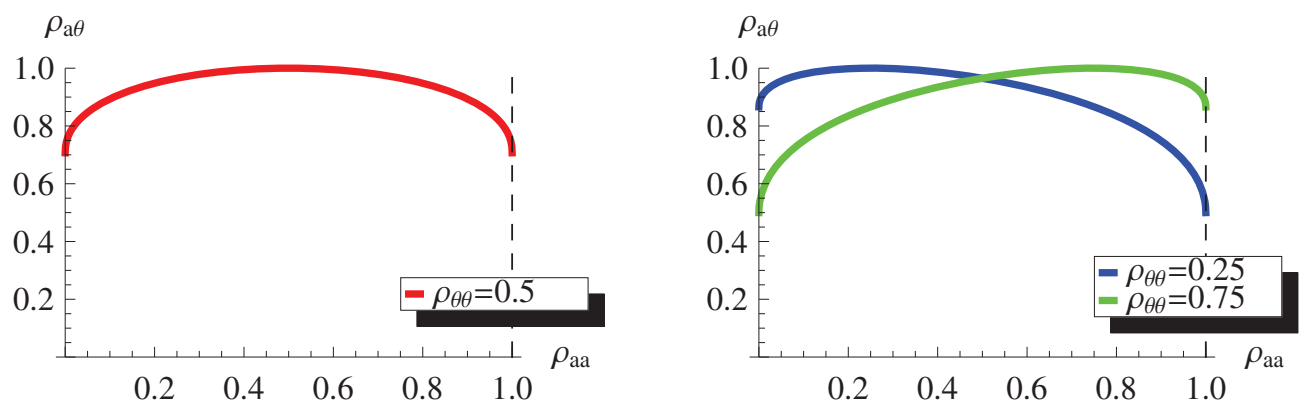

Figure 2: The Boundary of the Bayes Correlated Equilibrium Set

If the components $A$ and $\Delta a_{i}$ of the agent's individual action are completely explained by the components of the individual payoff shock, $\bar{\theta}$ and $\Delta \theta_{i}$, then the conditional variance of the action components, and a fortiori of the action itself, is equal to zero. ${ }^{4}$ In fact, the above conditional variances are equal to zero if the inequalities of Proposition 7 are satisfied as equalities. ${ }^{5}$

This restatement of the conditions for the boundary of the Bayes correlated equilibrium in terms of zero conditional variance provides a hint as to which information structures might attain the above boundary of the Bayes correlated equilibrium set as a Bayes Nash equilibrium. After all, the condition of zero conditional variance says that the action of each agent is completely explained by variations in the idiosyncratic and aggregate shock. But precisely this property was guaranteed by the noise free information structures that we considered in Section 3. Indeed, we can now provide an equivalence result akin to Proposition 8 , but this time specialized to the boundary of the Bayes correlated equilibrium set.

\section{Proposition 11 (Equivalence).}

The coefficients $\left(\rho_{a a}, \rho_{a \theta}, \rho_{a \phi}\right)$ form a boundary Bayes correlated equilibrium if and only if they form a noise free Bayes Nash equilibrium for some information structure $\lambda$.

The early restriction to noise free information structure in Section 3 may have appeared ad hoc. Proposition 11 establishes that the noise free information structures are economically significant in

\footnotetext{
${ }^{4}$ The matrix of conditional variances, (38), is well-defined only in the joint presence of idiosyncratic and aggregate shocks, that is for $\rho_{\theta \theta} \in(0,1)$. For the case of pure idiosyncratic or pure aggregate shocks, $\rho_{\theta \theta}=0$ or $\rho_{\theta \theta}=1$, one of the conditioning terms, $\bar{\theta}$ or $\Delta \theta_{i}$, has zero variance by definition.

${ }^{5}$ The negative root of the equality version of (34) also leads to the zero conditional variance. The resulting correlation coefficients could be generated by noise free information structures with $\lambda<0$. But the correlation coefficients are never part of the upper boundary of the Bayes correlated equilibrium set as Footnote 3 indicates.
} 
that they attain the maximally achievable equilibrium correlations among all possible information structures. Thus, the boundary of the Bayes correlated equilibria can be informationally decentralized by signals of the form (35) after imposing the requirement that $\sigma_{\varepsilon}=0$ and $\lambda \in[0,1]$, and hence precisely the one-dimensional noise free information structures investigated in Section 3.

A crucial implication of the characterization of the boundary in terms of the correlation coefficients is that if we seek to identify the equilibrium distribution that maximizes volatility or dispersion in the economy, then it is without loss of generality to focus on the boundary of the Bayes correlated equilibrium set. Moreover, by Proposition 11, this means that we can restrict attention to the class of one-dimensional noise free information structure.

Proposition 12 (Maximal Volatility and Dispersion).

Among all Bayes correlated equilibria, individual volatility, aggregate volatility, and dispersion are all maximized by a boundary Bayes correlated equilibrium.

More generally, the construction of the boundary suggests that any monotone function of the correlation coefficients $\left(\rho_{a a}, \rho_{a \theta}, \rho_{a \phi}\right)$ should achieve its maximum across all possible Bayes correlated equilibria somewhere on the boundary. This indicates that the noise free information structures remain the critical ones if we were to conduct a more comprehensive welfare analysis beyond the analysis of the second moments here. Notably, an auxiliary result for Proposition 12, Lemma 2 in the Appendix, establishes that any continuous function, $\psi$, with $\left(\rho_{a a}, \rho_{a \theta}, \rho_{a \phi}\right) \mapsto \psi\left(\rho_{a a}, \rho_{a \theta}, \rho_{a \phi}\right)$, that is strictly increasing in $\rho_{a \theta}$ and weakly increasing in $\rho_{a \phi}$, achieves its maximum in the set of all feasible Bayes correlated equilibria on the boundary of the set. As the conditions of Lemma 2 are silent about the correlation coefficient $\rho_{a a}$, we can accommodate strategic environments (and payoffs and associated objective functions) with either strategic substitutes or complements. Thus, we conclude that the special class of noise free information structures are indeed the relevant information structures even if we were to analyze a larger class of welfare functions.

In the discussion following Proposition 7, we argued that the moment restrictions remain necessary conditions even in the absence of any distributional assumptions of normality. Therefore, we can actually state a stronger version of Proposition 12. Suppose we maintain the assumption of normality in the payoff shocks, but neither do we require the normality in actions nor the joint normality in actions and shocks. Then, we would still have the result that the volatility is maximized by the noise free and normally distributed equilibria of Proposition 12, as the necessary boundary conditions of the Bayes correlated equilibria given by Proposition 7 are indeed attained by linear 
combinations of the idiosyncratic and the aggregate shocks. ${ }^{6}$

\section{Information Structures and Equilibrium Behavior}

We began our analysis with a class of specific information structures (noise free) and then established that these noise free information structures indeed form the boundary of equilibrium behavior with respect to all (symmetric normal) information structures. It is usual to assume some particular, but not noise free, information structure. We now ask how restrictive these commonly used classes of information structures are with respect to the entire set of feasible equilibrium behavior. Our purpose here is only to illustrate how assumptions about the information structure can be restrictive, rather than providing a comprehensive account of all information structure that appeared in the literature. Our representative examples illustrate subtle implications of restrictions on the information structure. The specific information structures that we study are a subset of the following three-dimensional information structures:

$$
S_{i} \triangleq\left\{s_{i}^{1}=\theta_{i}+\varepsilon_{i}^{1}, s_{i}^{2}=\bar{\theta}+\varepsilon_{i}^{2}, s_{i}^{3}=\bar{\theta}+\bar{\varepsilon}^{3}\right\}
$$

where $\varepsilon_{i}^{1}, \varepsilon_{i}^{2}$ are idiosyncratic noise terms and $\bar{\varepsilon}^{3}$ is a common noise term, all normally distributed, independent and with zero mean. This class of information structures appears in the analysis of Angeletos and Pavan [6] and is parameterized by three variables, namely the variances $\left(\sigma_{\varepsilon^{1}}^{2}, \sigma_{\varepsilon^{2}}^{2}, \sigma_{\varepsilon^{3}}^{2}\right)$ of the noise terms. We begin by characterizing the set of feasible correlations when agents only observe a noisy idiosyncratic signal of their payoff shock $\theta_{i}:^{7}$

$$
s_{i}^{1}=\theta_{i}+\varepsilon_{i}^{1},
$$

and thus we set $\sigma_{\varepsilon^{2}}^{2}=\sigma_{\varepsilon^{3}}^{2}=\infty$. This class of signals is frequently used in the literature on information sharing, see Vives [23] and Raith [18].

Proposition 13 (Noisy Signal of Payoff State).

A set of correlations $\left(\rho_{a a}, \rho_{a \theta}, \rho_{a \phi}\right)$ can be achieved as a Bayes Nash equilibrium with an information

\footnotetext{
${ }^{6}$ We conjecture that Proposition 12 holds even more generally in environments without normally distributed payoff shocks. But in the absence of normally distributed payoff shocks, the associated noise free information structure is likely to be a nonlinear, rather than linear, function of the shocks.

${ }^{7}$ We emphasize the fact that conditions (32) and (33) must hold for any signal structure. Thus, by characterizing the set of feasible correlations, we are characterizing the set of feasible outcomes.
} 


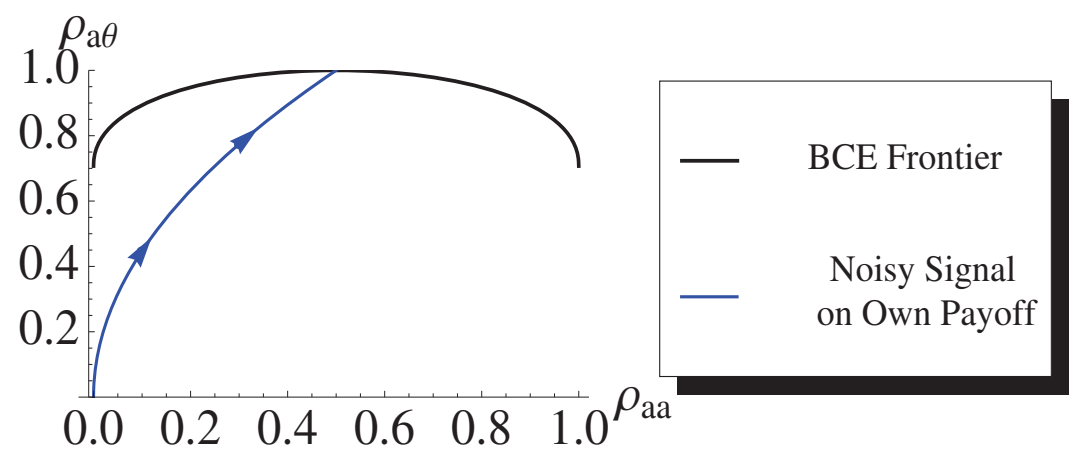

Figure 3: Feasible BNE correlation coefficients with noisy signals about payoff state $\theta_{i}$

structure $\left\{s_{i}^{1}\right\}_{i \in[0,1]}$, if and only if:

$$
\rho_{a \theta}=\sqrt{\frac{\rho_{a a}}{\rho_{\theta \theta}}} ; \quad \rho_{a \phi}=\rho_{a \theta} \rho_{\theta \theta} ; \quad \rho_{a a} \in\left[0, \rho_{\theta \theta}\right] .
$$

We observe that the set of feasible correlations when the agents receive only a one-dimensional signal of the form $s_{i}^{1}$ does not depend on the interaction parameter $r$. In Figure 3 we illustrate the locus of attainable correlations with information structures $\left\{s_{i}^{1}\right\}_{i \in[0,1]}$ for $\rho_{\theta \theta}=1 / 2$. The arrows point in the direction of greater precision (i.e., lower variance) of the error term. Notably, all the attainable equilibrium coefficients are below the frontier given by the Bayes correlated equilibria, except for a single point that is identified by zero noise, or $\sigma_{\varepsilon^{1}}^{2}=0$.

Next, we consider information structures in which each agent knows his own payoff shock, and thus we set $\sigma_{\varepsilon^{1}}^{2}=0$, but allow $\sigma_{\varepsilon^{2}}^{2}, \sigma_{\varepsilon^{3}}^{2} \in[0, \infty)$. That is, all possible outcomes that are consistent with each agent knowing at least $\theta_{i}$. Since each agent knows his own payoff shock, the residual uncertainty is with respect to the actions taken by the other players. The informational assumption that each agent knows his own payoff shock $\theta_{i}$ commonly appears in the macroeconomics literature. For example, Angeletos and La'O [2], [3] and Angeletos, Iovino, and La'O [1] consider models with idiosyncratic and aggregate shocks and imperfect information, but assume that each agent knows his own payoff shock $\theta_{i}$. In a model with idiosyncratic rather than aggregate interaction, Angeletos and La'O [4] analyze the impact of informational friction on aggregate fluctuations. Again, they assume that each agent knows his own payoff shock $\theta_{i}$, but is uncertain about the payoff shock $\theta_{j}$ of the trading partner $j$. Similarly, Lorenzoni [13] investigates the optimal monetary policy with dispersed information. He also considers a form of individual matching rather than aggregate 
interaction. The informational assumption common to all of these models is that every agent $i$ knows his own payoff shock $\theta_{i}$, and thus all uncertainty is purely strategic.

The characterization of the set of attainable equilibrium correlations is achieved in two steps. First, we describe the set of feasible action correlations $\rho_{a a}$. If each agent only knows his own payoff shock $\theta_{i}$, then the correlation $\rho_{a a}$ is equal to $\rho_{\theta \theta}$ as the actions of any two agents can only be correlated to the extent that their payoff shocks are correlated. By contrast, if the agents have complete information, then the correlation is given by $\rho_{a a}=\widehat{\rho}_{a a}$,

$$
\widehat{\rho}_{a a} \triangleq \frac{\rho_{\theta \theta}}{\left(1-\rho_{\theta \theta}\right)(1-r)^{2}+\rho_{\theta \theta}},
$$

where $\widehat{\rho}_{a a}$ is the correlation that is achieved by the information structure $\hat{\lambda}$ that recovers the equilibrium moments of the complete information Nash equilibrium, see (24).

We find that the set of feasible action correlations is always between these two quantities, $\rho_{\theta \theta}$ and $\widehat{\rho}_{a a}$, providing the lower and upper bound. If $r>0$, then the complete information bound is the upper bound, if $r<0$, it is the lower bound. For $r=0$ they coincide as $\theta_{i}$ is a sufficient statistic of the action taken by each agent under complete information.

Second, we describe the set of feasible correlations between action and individual payoff shock, $\rho_{a \theta}$, for any feasible $\rho_{a a}$. The set of feasible $\rho_{a \theta}$ is determined by two functions of $\rho_{a a}$, which provide the lower and upper bound for the feasible $\rho_{a \theta}$. We denote these functions by $\rho_{a \theta}^{i}\left(\rho_{a a}\right)$ and $\rho_{a \theta}^{c}\left(\rho_{a a}\right)$ as these bounds are achieved by information structures in which each agent knows his own payoff shock and receives a second signal, either an idiosyncratic signal of the aggregate shock $\bar{\theta}: s_{i}^{2} \triangleq \bar{\theta}+\varepsilon_{i}^{2}$ or a common signal of $\bar{\theta}: s_{i}^{3} \triangleq \bar{\theta}+\bar{\varepsilon}^{3}$.

Proposition 14 (Known Payoff State $\theta_{i}$ ).

A set of correlations $\left(\rho_{a a}, \rho_{a \theta}\right)$ can be induced by a linear Bayes Nash equilibrium in which each agent knows his payoff shock $\theta_{i}$ if and only if

$$
\rho_{a a} \in\left[\min \left\{\widehat{\rho}_{a a}, \rho_{\theta \theta}\right\}, \max \left\{\widehat{\rho}_{a a}, \rho_{\theta \theta}\right\}\right]
$$

and for any $\rho_{a a}$ satisfying (42):

$$
\rho_{a \theta} \in\left[\min \left\{\rho_{a \theta}^{c}\left(\rho_{a a}\right), \rho_{a \theta}^{i}\left(\rho_{a a}\right)\right\}, \max \left\{\rho_{a \theta}^{c}\left(\rho_{a a}\right), \rho_{a \theta}^{i}\left(\rho_{a a}\right)\right\}\right] .
$$

In Figure 4, we illustrate the Bayes Nash equilibrium set for different values of $r$ for a given correlation $\rho_{\theta \theta}=1 / 2$. Each interaction value $r$ is represented by a differently colored pair of lower and upper bounds. For each value of $r$, the entire set of Bayes Nash equilibria is given by the area enclosed by the lower and upper bounds. Notably, the bounds $\rho_{a \theta}^{c}\left(\rho_{a a}\right)$ and $\rho_{a \theta}^{i}\left(\rho_{a a}\right)$ intersect in 
two points, corresponding to each agent knowing his payoff shock $\theta_{i}$ only (at $\rho_{a a}=\rho_{\theta \theta}=1 / 2$ ) and to complete information, at the low or high end of $\rho_{a a}$ depending on the nature of the interaction, respectively. In fact these, and only these, two points, are also boundary points of the unrestricted set of Bayes correlated equilibria. When $r \geq 0$, the upper bound is given by a signal with an idiosyncratic error term, $s_{i}^{2}$, while the lower bound is given by a signal with a common error term, $s_{i}^{3}$, and conversely for $r \leq 0$.

Thus, if each agent knows at least his own payoff shock, then we observe a dramatic reduction in the set of feasible Bayes Nash equilibria. Notably, every element, with the exception of the information structures mentioned in the above paragraph, are in the interior of the unrestricted set of Bayes correlated equilibria. Moreover, the nature of the interaction has a profound impact on the set of correlations $\left(\rho_{a a}, \rho_{a \theta}\right)$ that can arise in equilibrium, both in terms of its size as well as its location in the unit square.

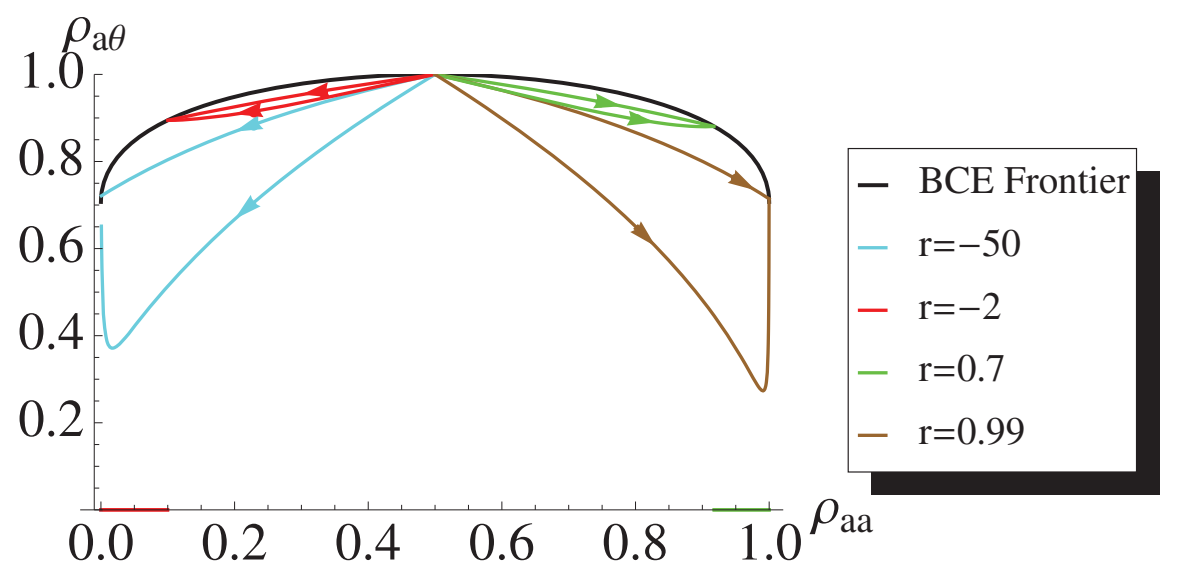

Figure 4: Boundary of the set of feasible correlations when agents know own payoff $\left(\rho_{\theta \theta}=1 / 2\right)$

If each agent $i$ is assumed to know his payoff shock $\theta_{i}$, then we can restate the best response condition (4) with respect to $a_{i}$ after the following change of variables in terms of deviations from the payoff shock:

$$
\tilde{a}_{i} \triangleq a_{i}-\theta_{i}, \quad \widetilde{A} \triangleq A-\bar{\theta} .
$$

The best response condition (4) in terms of $a_{i}$ reduces to the following best response condition in terms of $\tilde{a}_{i}$ :

$$
\tilde{a}_{i}=r \mathbb{E}\left[\bar{\theta} \mid \theta_{i}, \mathcal{I}_{i}\right]+r \mathbb{E}\left[\widetilde{A} \mid \theta_{i}, \mathcal{I}_{i}\right]
$$


where $\mathcal{I}_{i}$ is any information agent $i$ gets beyond knowing $\theta_{i}$. The resulting best response condition is now isomorphic to one where there are only aggregate shocks, and where the payoff shock and average action receive the same weight in the best response condition of the individual agent. This provides a distinct intuition on the strong restrictions on behavior that arise from imposing that agents know their own payoff shock as stated in Proposition 14.

In the supplemental online material, we analyze a third subset of information structure, in which, besides the noisy signal $\left\{s_{i}^{1}\right\}_{i \in[0,1]}$, each player also knows the aggregate shock $\bar{\theta}$, thus $\sigma_{\varepsilon^{2}}^{2}=\sigma_{\varepsilon^{3}}^{2}=0$. Although a priori this may not seem like an information structure that would arise exogenously, it is the information structure that arises when agents receive endogenous information on the average action taken by other players, such as in a rational expectations equilibrium with a continuum of sellers as studied by Vives [25] or Bergemann, Heumann, and Morris [7]. In the supplemental online material, we characterize the entire set of equilibrium correlations that can be achieved with the three-dimensional structures defined by (39). Surprisingly then the above class of three-dimensional information structures $S_{i}$ fails to decentralize the entire set of Bayes correlated equilibria. By contrast, in the case of pure aggregate shocks Bergemann and Morris [8] show that any Bayes correlated equilibrium can be decentralized by considering a private and a public signal of the payoff shock, namely $s_{i}^{1}=\theta_{i}+\varepsilon_{i}^{1}$ and $s_{i}^{3}=\bar{\theta}+\bar{\varepsilon}^{3}$.

\section{Discussion}

We conclude by discussing the relevance of the current analysis to environments with heterogeneous rather than aggregate interaction. We end by relating our analysis to the large literature on information sharing among firms and suggest how the current tools might yield new results there as well.

Beyond Aggregate Interaction. We deliberately restricted our analysis to an environment with aggregate interaction. Every agent formed a best response against the average of the population. Yet, within the linear quadratic framework, it appears feasible to extend the analysis to much richer interaction structures, such as pairwise interaction or even general network interaction structures. In the macroeconomics literature, models of heterogenous interactions have appeared prominently, for example, in Lorenzoni [13] and Angeletos and La'O [4]. Notably, these models of pairwise interaction assume that each agent knows his own payoff shock $\theta_{i}$ but is still uncertain about the payoff shocks of other agents. As each agent $i$ knows his own payoff shock $\theta_{i}$, there is no payoff uncertainty 
anymore and so the residual uncertainty is all about the strategic uncertainty, namely the action of the other agent. 8

Interestingly, even if we were interested in strategic uncertainty in the absence of payoff uncertainty, the noise free information structures remain of central importance for the aggregate behavior. To see this, consider a simple model of pairwise interaction as in Angeletos and La'O [4]. We assume there is pairwise matching between $i$ and $j$ and that agents interact with their partner, $r_{a}$, as well as with the aggregate population, $r_{A}$. Thus, the first order condition of agent $i$ 's problem when he is matched with $j$ is given by:

$$
a_{i}=\mathbb{E}\left[\theta_{i} \mid \theta_{i}, \mathcal{I}_{i}\right]+r_{a} \mathbb{E}\left[a_{j} \mid \theta_{i}, \mathcal{I}_{i}\right]+r_{A} \mathbb{E}\left[A \mid \theta_{i}, \mathcal{I}_{i}\right]
$$

If we make the same change of variables as earlier in (43), so that we express the choice variables in terms of their deviation from the payoff shock: $\tilde{a}_{i} \triangleq a_{i}-\theta_{i}, \quad \widetilde{A} \triangleq A-\bar{\theta}$, then the associated first order conditions are given by:

$$
\tilde{a}_{i}=r_{a} \mathbb{E}\left[\Delta \theta_{j} \mid \theta_{i}, \mathcal{I}_{i}\right]+r_{A} \mathbb{E}\left[\bar{\theta} \mid \theta_{i}, \mathcal{I}_{i}\right]+r_{a} \mathbb{E}\left[\widetilde{a}_{j} \mid \theta_{i}, \mathcal{I}_{i}\right]+r_{A} \mathbb{E}\left[\widetilde{A} \mid \theta_{i}, \mathcal{I}_{i}\right] .
$$

Thus, we have a similar model as the one we have been studying so far, but with some differences. First, agents have some prior information on $\bar{\theta}$ which comes from knowing $\theta_{i}$. Second, the size of the shocks $\Delta \theta_{j}$ and $\bar{\theta}$ are scaled by $r_{a}$ and $r_{A}$, respectively, in the first order conditions. Besides these differences, a model with heterogeneous interaction in which each agent knows his own payoff shock is almost identical to our original model. Namely, each agent's uncertainty is still two-dimensional, with an aggregate and an idiosyncratic shock (equal to $\bar{\theta}$ and $\Delta \theta_{j}$ respectively). Thus, we see that even if we were interested in strategic uncertainty in the absence of fundamental uncertainty, the same basic intuitions and ideas would still apply. A key factor to consider would be to see how the signal leads each agent to be confuses about $\Delta \theta_{j}$ and $\bar{\theta}$. And as before, the confounding of the information would lead to overreaction and underreaction to some of these fundamentals, respectively. Thus, the pairwise interaction, or any other richer interaction structure, enriches the set of feasible outcomes and partially reverses the restrictions that come with the informational assumptions of knowing the individual payoff states that we earlier established in Proposition 14.

Information Sharing. We described the impact that the private information structure has on the second moments of the economy, in particular the volatility of the aggregate outcome. Naturally,

\footnotetext{
${ }^{8}$ We thank our discussant, Marios Angeletos, for emphasizing the importance of the distinct contribution of each source of uncertainty to the aggregate volatility.
} 


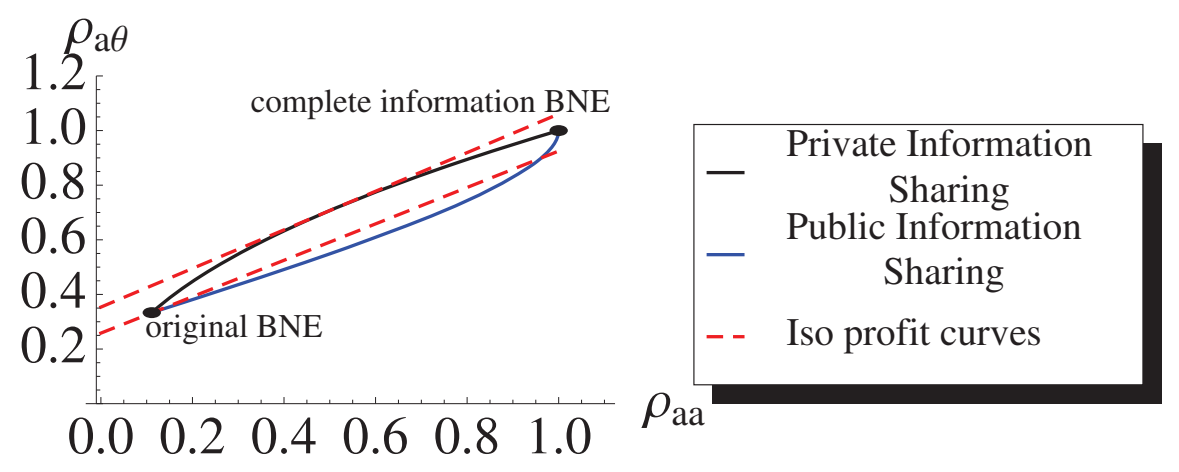

Figure 5: Information Sharing under Public and Private Disclosure Rules

we could expand the analysis to functions of the (second) moments of the economy. In the large literature on information sharing among firms, pioneered in work by Novshek and Sonnenschein [17], Clarke [10] and Vives [22], the expected profit function of the individual firm is a function of the volatility both of the individual and the aggregate outcome. In this class of models which is presented in a very general framework by Raith [18] and surveyed by Vives [24], each firm receives a private signal about a source of uncertainty, say a demand or cost shock. The central question is under which conditions the firms have an incentive to commit ex-ante to an agreement to share information in some form. The present analysis of the impact of information structures on the set of feasible correlations suggests novel insights into the nature of optimal information sharing policies.

We briefly illustrate this within a competitive equilibrium with a continuum of producers, each one of them with a quadratic cost of production $c\left(a_{i}\right)=a_{i}^{2} / 2$, and facing a linear inverse demand function dependent on the aggregate demand shock $\bar{\theta}$ and the aggregate supply $A$ : $p(\bar{\theta}, A)=\bar{\theta}+r A$, so that the resulting best response function is again given by (4). ${ }^{9}$ We can depict the iso-profit curve $\bar{\pi}$ defined implicitly by a constant expected profit $\bar{\pi}$ of the representative firm, $\bar{\pi} \triangleq \mathbb{E}\left[a_{i} p-\frac{1}{2} a_{i}^{2}\right]$, in the space of the correlation coefficients $\left(\rho_{a a}, \rho_{a \theta}\right)$. The iso-profit curve $\bar{\pi}$ can be shown to be linear in $\rho_{a a}$, as indicated by the red dashed line in Figure 5, and the slope is determined by the responsiveness $r$ of the price to supply.

The maximal correlation $\rho_{a \theta}$ that is achievable with disclosure of a common signal, denoted

\footnotetext{
${ }^{9}$ The restriction to the pure aggregate shock environment, $\theta_{i}=\bar{\theta}$, allows us to directly use arguments in Bergemann and Morris [8], in particular Proposition 8, but the insights naturally extend to the environment with idiosyncratic and aggregate shocks.
} 
earlier in Proposition 14 by $\rho_{a \theta}^{c}\left(\rho_{a a}\right)$, is convex in $\rho_{a a}$, whereas the maximal correlation achievable with disclosure of an idiosyncratic signal is given by $\rho_{a \theta}^{i}\left(\rho_{a a}\right) \triangleq \sqrt{\rho_{a a}}$, and is concave in $\rho_{a a}$. In fact with aggregate shocks only, the idiosyncratic signals $s_{i}^{1}=\bar{\theta}+\varepsilon_{i}$ trace out the entire boundary of the Bayes correlated equilibrium coefficients, as illustrated in Figure 5. We therefore can conclude that the optimal disclosure policy with a public signal is either zero or complete disclosure, which was a central finding in Kirby [12], Vives [23] and Raith [18]. ${ }^{10}$ By contrast, the optimal disclosure policy of a private signal depends on $r$ and can be noisy. The iso-profit curve generates a linear trade-off in the correlations of the individual supply decision $a_{i}$ and the demand shock $\bar{\theta}$. A better match with the level of aggregate demand increases profit, but a better match with the supply of the other firms decreases the profit. With public disclosure of a noisy signal $s_{i}$, the convexity in the trade-off leads to either zero disclosure or complete disclosure of the aggregate information. With private disclosure, the trade-off is resolved in favor of a better match with the demand shock without an undue increase in the correlation of the supply decisions. Thus, we find that the industry-wide preferred disclosure policy frequently involves partial disclosure of information, by which disclosure is noisy and idiosyncratic, as opposed to the bang-bang solution that was previously obtained in the literature under the (implicit) restriction to public disclosure policies. Thus we find that a common and hence perfectly correlated disclosure policy is (always) weakly and (sometimes) strictly dominated by a private and hence imperfectly correlated disclosure policy.

We can rephrase this insight in terms of the macroeconomic language. An idiosyncratic "sentiment" shock may be needed to generate the largest individual volatility in the aggregate shock environment, just as a common "sentiment" shocks is necessary to generate the largest volatility in the idiosyncratic shock environment of Angeletos and La'O [4]. The analysis in Section 7 suggests that the above results for the pure aggregate shock environment (and similarly for the pure idiosyncratic shock) extend to the general environment with idiosyncratic as well as aggregate shocks. We leave a more comprehensive analysis for future research.

\footnotetext{
${ }^{10}$ In Section 8.4 of Vives [24], the design of the optimal information sharing policy in a large market with a continuum of agents is posed as the problem of a mediator who elicits and then transmits the collected information to the agents. The analysis is thus close to the present perspective of the Bayes correlated equilibrium, but also restricts the transmission policy to public signals, and hence leads to the same conclusion as the above mentioned literature.
} 


\section{Appendix A}

The Appendix A collects the omitted proofs from the main body of the text. The supplementary online material contains the Appendices B and C, which have additional results regarding the restrictions that information structures impose on the equilibrium behavior, complementing the results of Section 7 of the main paper.

Proof of Proposition 1. Since the actions of players must be measurable with respect to $s_{i}$, in any linear strategy the actions of players must be given by $a_{i}=w(\lambda) s_{i}+\nu$, where $\nu$ and $w(\lambda)$ are constants. Thus $A=w(\lambda)((1-\lambda) \bar{\theta})+\nu$. Thus, we must have that:

$$
a_{i}=w(\lambda) s_{i}+\nu=\mathbb{E}\left[r(w(\lambda)((1-\lambda) \bar{\theta})+\nu)+\theta_{i} \mid s_{i}\right] .
$$

By taking expectations and using the law of iterated expectations, we get:

$$
w(\lambda)(1-\lambda) \mu_{\theta}+\nu=r w(\lambda)\left((1-\lambda) \mu_{\theta}+\nu\right)+\mu_{\theta}
$$

Using that $\mu_{\theta}=0$, we get that $\nu=0$. Thus, we know that $a_{i}=w(\lambda)\left((1-\lambda) \bar{\theta}+\lambda \Delta \theta_{i}\right)$ and $A=w(\lambda)(1-\lambda) \bar{\theta}$. Multiplying by $a_{i}$ we get: $a_{i}^{2}=\mathbb{E}\left[r A a_{i}+\theta_{i} a_{i} \mid s_{i}\right]$, and appealing to the law of iterated expectations we get:

$$
w(\lambda)\left((1-r)(1-\lambda)^{2} \rho_{\theta \theta}+\lambda^{2}\left(1-\rho_{\theta \theta}\right)\right)=\left((1-\lambda) \rho_{\theta \theta}+\lambda\left(1-\rho_{\theta \theta}\right)\right),
$$

and solving for $w(\lambda)$ yields the expression in (11). The uniqueness of the Bayes Nash equilibrium is established in Ui and Yoshizawa [19].

Proof of Proposition 2. By using the law of iterated expectations we obtain $\mu_{a}=\mu_{A}=$ $\mu_{\theta} /(1-r)$. We can compute the variance and covariances by using (10) and (11). It is easy to see that:

$$
\operatorname{var}\left(a_{i}\right)=\sigma_{a}^{2}=w(\lambda)^{2} \operatorname{var}\left(s_{i}\right)=w(\lambda)^{2}\left((1-\lambda)^{2} \rho_{\theta \theta}+\lambda^{2}\left(1-\rho_{\theta \theta}\right)\right) \sigma_{\theta}^{2},
$$

thus we get (12). Similarly, we obtain:

$\operatorname{var}(A)=\operatorname{cov}\left(a_{i}, a_{j}\right)=\rho_{a a} \sigma_{a}^{2}=w(\lambda)^{2} \mathbb{E}\left[\left((1-\lambda) \bar{\theta}+\lambda \Delta \theta_{i}\right)\left((1-\lambda) \bar{\theta}+\lambda \Delta \theta_{j}\right)\right]=w(\lambda)^{2}(1-\lambda)^{2} \rho_{\theta \theta} \sigma_{\theta}^{2}$,

and

$$
\operatorname{cov}\left(a_{i}, \theta_{i}\right)=\rho_{a \theta} \sigma_{a} \sigma_{\theta}=w(\lambda) \mathbb{E}\left[\left((1-\lambda) \bar{\theta}+\lambda \Delta \theta_{i}\right) \theta_{i}\right]=w(\lambda)\left((1-\lambda) \rho_{\theta \theta}+\lambda\left(1-\rho_{\theta \theta}\right)\right) \sigma_{\theta}^{2},
$$

which establishes the result. 
Proof of Proposition 3. The result follows directly from solving the following maximization problem:

$$
\max _{\lambda}\left(\frac{(1-\lambda) \rho_{\theta \theta}+\lambda\left(1-\rho_{\theta \theta}\right)}{(1-\lambda)^{2} \rho_{\theta \theta}+\lambda^{2}\left(1-\rho_{\theta \theta}\right)}\right)^{2}(1-\lambda)^{2} \rho_{\theta \theta} .
$$

The solution (17) follows from Proposition 6.

Proof of Proposition 4. Given a noise free equilibrium parametrized by $\lambda$ we have that:

$$
\begin{gathered}
\operatorname{cov}\left(a_{i}, \bar{\theta}\right)=w(\lambda)(1-\lambda) \bar{\theta}=\frac{\left((1-\lambda) \rho_{\theta \theta}+\lambda\left(1-\rho_{\theta \theta}\right)\right)}{\left((1-r)(1-\lambda)^{2} \rho_{\theta \theta}+\lambda^{2}\left(1-\rho_{\theta \theta}\right)\right)}(1-\lambda) \bar{\theta}, \\
\operatorname{cov}\left(a_{i}, \Delta \theta_{i}\right)=w(\lambda) \lambda \Delta \theta_{i}=\frac{\left((1-\lambda) \rho_{\theta \theta}+\lambda\left(1-\rho_{\theta \theta}\right)\right)}{\left((1-r)(1-\lambda)^{2} \rho_{\theta \theta}+\lambda^{2}\left(1-\rho_{\theta \theta}\right)\right)} \lambda \Delta \theta_{i} .
\end{gathered}
$$

But, note that if $\lambda<\widehat{\lambda}$, then $\frac{\lambda}{(1-r)}<(1-\lambda)$, but then

$$
\begin{aligned}
\operatorname{cov}\left(a_{i}, \Delta \theta_{i}\right) & =\frac{\left((1-\lambda) \rho_{\theta \theta} \lambda+\lambda^{2}\left(1-\rho_{\theta \theta}\right)\right)}{\left((1-r)(1-\lambda)^{2} \rho_{\theta \theta}+\lambda^{2}\left(1-\rho_{\theta \theta}\right)\right)} \Delta \theta_{i} \\
& \geq \frac{\left((1-\lambda)^{2}(1-r) \rho_{\theta \theta}+\lambda^{2}\left(1-\rho_{\theta \theta}\right)\right)}{\left((1-r)(1-\lambda)^{2} \rho_{\theta \theta}+\lambda^{2}\left(1-\rho_{\theta \theta}\right)\right)}=1
\end{aligned}
$$

with strict inequality if $\lambda>\widehat{\lambda}$. Thus, the response to the idiosyncratic shock is greater than in the complete information equilibrium if $\lambda \in(\widehat{\lambda}, 1)$. For the second part we repeat the same argument. Note that if $\lambda<\hat{\lambda}$, then $\lambda<(1-\lambda)(1-r)$, but then:

$$
\begin{aligned}
\operatorname{cov}\left(a_{i}, \bar{\theta}\right) & =\frac{\left((1-\lambda)^{2} \rho_{\theta \theta}+(1-\lambda) \lambda\left(1-\rho_{\theta \theta}\right)\right)}{\left((1-r)(1-\lambda)^{2} \rho_{\theta \theta}+\lambda^{2}\left(1-\rho_{\theta \theta}\right)\right)} \bar{\theta} \\
& \geq \frac{1}{1-r} \frac{\left((1-\lambda)^{2}(1-r) \rho_{\theta \theta}+\lambda^{2}\left(1-\rho_{\theta \theta}\right)\right)}{\left((1-r)(1-\lambda)^{2} \rho_{\theta \theta}+\lambda^{2}\left(1-\rho_{\theta \theta}\right)\right)}=\frac{1}{1-r},
\end{aligned}
$$

with strict inequality if $\lambda<\widehat{\lambda}$.

Proof of Proposition 5. The comparative statics with respect to the argmax are shown by proving that the quantities have a unique maximum, which is interior, and then use the sign of the cross derivatives (the derivative with respect to $\lambda$ and $r$ ). The ordering of the information structures that maximizes the different second moments is proved by comparing the derivatives.

(2.) We begin by rewriting the individual variance, and using (12) we can write it in terms of $\lambda$ :

$$
\begin{aligned}
\sigma_{a}^{2} & =\left(\frac{\left((1-\lambda) \rho_{\theta \theta}+\lambda\left(1-\rho_{\theta \theta}\right)\right)}{\left((1-r)(1-\lambda)^{2} \rho_{\theta \theta}+\lambda^{2}\left(1-\rho_{\theta \theta}\right)\right)}\right)^{2}\left((1-\lambda)^{2} \rho_{\theta \theta}+\lambda^{2}\left(1-\rho_{\theta \theta}\right)\right) \sigma_{\theta}^{2} \\
& =\rho_{\theta \theta} \frac{(1+y x)^{2}}{\left((1-r)+x^{2}\right)^{2}}\left(1+x^{2}\right) \sigma_{\theta}^{2}
\end{aligned}
$$


where

$$
x \triangleq \frac{\sqrt{\left(1-\rho_{\theta \theta}\right)} \lambda}{\sqrt{\rho_{\theta \theta}}(1-\lambda)}, \quad y \triangleq \frac{\sqrt{1-\rho_{\theta \theta}}}{\sqrt{\rho_{\theta \theta}}} .
$$

Note that $x$ is strictly increasing in $\lambda$, and if $\lambda \in[0,1]$ then $x \in[0, \infty]$, and thus maximizing with respect to $x \in[0, \infty]$ is equivalent to maximizing with respect to $\lambda \in[0,1]$. Finding the derivative we get:

$$
\frac{\partial \sigma_{a}^{2}}{\partial x}=-\frac{2(x y+1)\left(x^{3}+(2 r-1) y x^{2}+(r+1) x-(1-r) y\right)}{\left(x^{2}+1-r\right)^{3}} \sigma_{\theta}^{2} .
$$

It is easy to see that $\frac{d \sigma_{a}^{2}}{d x}$ is positive at $x=0$ and negative if we take a $x$ large enough, and thus the maximum must be in $x \in(0, \infty)$. We would like to show that the polynomial:

$$
\left(x^{3}+(2 r-1) y x^{2}+(r+1) x-(1-r) y\right),
$$

has a unique root in $x \in(0, \infty)$. If $r<-1$, then the function is increasing in $x$ and has a negative value at $x=0$, thus it has a unique root. If $x>1 / 2$, then the function is negative and decreasing at $x$. Since it is a cubic polynomial and the term next to $x^{3}$ is positive, it must have a unique positive root. For $r \in[-1,1 / 2]$ we define the determinant of the cubic equation:

$$
\Delta=18 a b c d-4 b^{3} d+b^{2} c^{2}-4 a c^{3}-27 a^{2} d^{2} .
$$

We know that if $\Delta<0$ then the polynomial has a unique root. Replacing by the respective values of the cubic polynomial we get:

$$
\left.\Delta=4 y^{4}(2 r-1)^{3}(1-r)+y^{2}\left((2 r-1)^{2}(1+r)^{2}-18\left(1-r^{2}\right)(2 r-1)-27(1-r)^{2}\right)\right)-4(1+r)^{3},
$$

using the fact that for $r \in[-1,1 / 2]$ we have that $(2 r-1) \leq 0$ and $1+r \geq 0$, we know that the term with $y^{4}$ and without $y$ are negative. We just need to check the term with $y^{2}$, but this is also negative for $r \in[-1,1 / 2]$. Thus, $\Delta<0$, and thus for $r \in[-1,1 / 2]$ the polynomial has a unique root.

Thus, we have that there exists a unique $\lambda$ that maximizes $\sigma_{a}^{2}$. Finally, we have that:

$$
\frac{\partial \sigma_{a}^{2}}{\partial r}=2 \frac{(1-\lambda)^{2} \rho_{\theta \theta}}{\left((1-r)(1-\lambda)^{2} \rho_{\theta \theta}+\lambda^{2}\left(1-\rho_{\theta \theta}\right)\right)} \sigma_{a}^{2} .
$$

Note that

$$
\frac{\partial}{\partial \lambda} \frac{(1-\lambda)^{2} \rho_{\theta \theta}}{\left((1-r)(1-\lambda)^{2} \rho_{\theta \theta}+\lambda^{2}\left(1-\rho_{\theta \theta}\right)\right)}<0,
$$


and thus at the maximum:

$$
\frac{\partial^{2} \sigma_{a}^{2}}{\partial r \partial \lambda}=2 \sigma_{a}^{2} \frac{\partial}{\partial \lambda} \frac{(1-\lambda)^{2} \rho_{\theta \theta}}{\left((1-r)(1-\lambda)^{2} \rho_{\theta \theta}+\lambda^{2}\left(1-\rho_{\theta \theta}\right)\right)}<0
$$

and thus $\operatorname{argmax}_{\lambda} \sigma_{a}^{2}$ is decreasing in $r$.

Next, we consider the aggregate variance $\rho_{a a} \sigma_{a}^{2}$, and write it in terms of $\lambda$ :

$$
\rho_{a a} \sigma_{a}^{2}=\left(\frac{\left((1-\lambda) \rho_{\theta \theta}+\lambda\left(1-\rho_{\theta \theta}\right)\right)}{\left((1-r)(1-\lambda)^{2} \rho_{\theta \theta}+\lambda^{2}\left(1-\rho_{\theta \theta}\right)\right)}\right)^{2}(1-\lambda)^{2} \rho_{\theta \theta} \sigma_{\theta}^{2}=\rho_{\theta \theta} \frac{(1+y x)^{2}}{\left((1-r)+x^{2}\right)^{2}} \sigma_{\theta}^{2},
$$

where $x$ and $y$ are defined as in (46). Maximizing with respect to $x \in[0, \infty]$ is equivalent to maximizing with respect to $\lambda \in[0,1]$. Finding the derivative we get:

$$
\frac{\partial \rho_{a a} \sigma_{a}^{2}}{\partial x}=-\frac{2(x y+1)\left(2 x+\left(x^{2}+r-1\right) y\right)}{\left(x^{2}+1-r\right)^{3}} \sigma_{\theta}^{2} .
$$

Again, we have that $\left(2 x+\left(x^{2}+r-1\right) y\right)$ has a unique root in $(0, \infty)$ Thus, we have that there exists a unique $\lambda$ that maximizes $\rho_{a a} \sigma_{a}^{2}$. Finally, we have that:

$$
\frac{\partial \rho_{a a} \sigma_{a}^{2}}{\partial r}=2 \frac{(1-\lambda)^{2} \rho_{\theta \theta}}{\left((1-r)(1-\lambda)^{2} \rho_{\theta \theta}+\lambda^{2}\left(1-\rho_{\theta \theta}\right)\right)} \rho_{a a} \sigma_{a}^{2} .
$$

Note that,

$$
\frac{\partial}{\partial \lambda} \frac{(1-\lambda)^{2} \rho_{\theta \theta}}{\left((1-r)(1-\lambda)^{2} \rho_{\theta \theta}+\lambda^{2}\left(1-\rho_{\theta \theta}\right)\right)}<0,
$$

and thus at the maximum $\frac{\partial^{2} \sigma_{a}^{2}}{\partial r \partial \lambda}<0$, and thus $\operatorname{argmax}_{\lambda} \rho_{a a} \sigma_{a}^{2}$ is decreasing in $r$.

Finally, we consider the dispersion, $\left(1-\rho_{a a}\right) \sigma_{a}^{2}$, expressed in terms of $\lambda$ :

$$
\begin{aligned}
\left(1-\rho_{a a}\right) \sigma_{a}^{2} & =\left(\frac{\left((1-\lambda) \rho_{\theta \theta}+\lambda\left(1-\rho_{\theta \theta}\right)\right)}{\left((1-r)(1-\lambda)^{2} \rho_{\theta \theta}+\lambda^{2}\left(1-\rho_{\theta \theta}\right)\right)}\right)^{2} \lambda^{2}\left(1-\rho_{\theta \theta}\right) \sigma_{\theta}^{2} \\
& =\rho_{\theta \theta} \frac{(1+y x)^{2}}{\left((1-r)+x^{2}\right)^{2}} x^{2} \sigma_{\theta}^{2}
\end{aligned}
$$

where $x$ and $y$ are defined in (46). As before, maximizing with respect to $x \in[0, \infty]$ is equivalent to maximizing with respect to $\lambda \in[0,1]$. Finding the derivative we get:

$$
\frac{\partial\left(1-\rho_{a a}\right) \sigma_{a}^{2}}{\partial x}=-\frac{2 x(x y+1)\left(x^{2}+2(r-1) y x+r-1\right)}{\left(x^{2}+1-r\right)^{3}} \sigma_{\theta}^{2} .
$$

Again, we have that $\left(x^{2}+2(r-1) y x+r-1\right)$ has a unique root in $(0, \infty)$ Thus, there exists a unique $\lambda$ that maximizes $\left(1-\rho_{a a}\right) \sigma_{a}^{2}$. Finally, we have that:

$$
\frac{\partial\left(1-\rho_{a a}\right) \sigma_{a}^{2}}{\partial r}=2 \frac{(1-\lambda)^{2} \rho_{\theta \theta}}{\left((1-r)(1-\lambda)^{2} \rho_{\theta \theta}+\lambda^{2}\left(1-\rho_{\theta \theta}\right)\right)}\left(1-\rho_{a a}\right) \sigma_{a}^{2} .
$$


Note that

$$
\frac{\partial}{\partial \lambda} \frac{(1-\lambda)^{2} \rho_{\theta \theta}}{\left((1-r)(1-\lambda)^{2} \rho_{\theta \theta}+\lambda^{2}\left(1-\rho_{\theta \theta}\right)\right)}<0,
$$

and thus at the maximum $\frac{\partial^{2} \sigma_{a}^{2}}{\partial r \partial \lambda}<0$, and thus $\operatorname{argmax}_{\lambda}\left(1-\rho_{a a}\right) \sigma_{a}^{2}$ is decreasing in $r$.

(1.) Finally, we want to show that $\operatorname{argmax}_{\lambda}\left(1-\rho_{a a}\right) \sigma_{a}^{2}>\operatorname{argmax}_{\lambda} \sigma_{a}^{2}>\operatorname{argmax}_{\lambda} \rho_{a a} \sigma_{a}^{2}$. These inequalities follows from comparing the derivatives of $\left(1-\rho_{a a}\right) \sigma_{a}^{2}, \sigma_{a}^{2}$ and $\rho_{a a} \sigma_{a}^{2}$ with respect to $\lambda$ (or equivalently $x$ ). It is easy to see that:

$$
\frac{\partial \log \left(1-\rho_{a a}\right) \sigma_{a}^{2}}{\partial x}<\frac{\partial \log \sigma_{a}^{2}}{\partial x}<\frac{\partial \log \rho_{a a} \sigma_{a}^{2}}{\partial x} .
$$

Since the derivatives satisfy the previous inequalities, and the quantities have a unique maximum, the argument of the maximum must also satisfy the same inequalities.

(3.) The comparative static results with respect to the maximum follow directly from the envelope theorem.

Proof of Proposition 6. We first solve for $\max _{\lambda}\left\{\rho_{a a} \sigma_{a}^{2}\right\}$. By setting (48) equal to 0 , we have that the aggregate volatility is maximized at,

$$
x=\frac{\sqrt{1+y^{2}(1-r)}-1}{y} .
$$

In terms of the original variables this can be written as follows:

$$
\lambda=\frac{\rho_{\theta \theta}\left(\sqrt{\frac{1-r}{\rho_{\theta \theta}}+r}+r-2\right)}{(r-4) \rho_{\theta \theta}+1} .
$$

Substituting the solution in (47) and using the definitions of $x$ and $y$ we get that the maximum volatility is equal to:

$$
\frac{\sigma_{\theta}^{2}\left(1-\rho_{\theta \theta}\right)^{2}}{\left.4\left(\sqrt{\rho_{\theta \theta}}-\sqrt{\rho_{\theta \theta}+(1-r)\left(1-\rho_{\theta \theta}\right.}\right)\right)^{2}} .
$$

Using the definition of $\sigma_{\bar{\theta}}$ and $\sigma_{\theta}^{2}$ we get (26). Note that by imposing $r=0$ we also get (17) and (18).

Proof of Corollary 3. It follows directly from (26) that:

$$
\lim _{\sigma_{\bar{\theta}}^{2} \rightarrow 0} \max _{\lambda}\left\{\rho_{a a} \sigma_{a}^{2}\right\}=\frac{\sigma_{\theta}^{2}}{4(1-r)} .
$$

Thus, we are only left with proving that,

$$
\lim _{\sigma_{\Delta \theta_{i}}^{2} \rightarrow 0} \max _{\lambda}\left\{\rho_{a a} \sigma_{a}^{2}\right\}=\sigma_{\bar{\theta}}^{2} /(1-r)^{2} .
$$


The limit can be easily calculated using L'Hopital's rule. That is, just note that as $\sigma_{\Delta \theta_{i}}^{2} \rightarrow 0$ we have that:

$$
4\left(\sigma_{\bar{\theta}}-\sqrt{\sigma_{\bar{\theta}}^{2}+(1-r) \sigma_{\Delta \theta_{i}}^{2}}\right)^{2} \approx \sigma_{\Delta \theta_{i}}^{4}(1-r)^{2} / \sigma_{\bar{\theta}}^{2}+o\left(\sigma_{\Delta \theta_{i}}^{6}\right),
$$

and hence we get the result.

Proof of Lemma 1. We need to prove that given the assumption of symmetry, the parameters $\left(\mu_{a}, \rho_{a a}, \rho_{a \theta}, \rho_{a \phi}, \sigma_{a}\right)$ are sufficient to characterize the distribution of the random variables $\left(\theta_{i}, \bar{\theta}, a_{i}, A\right)$. Clearly, we have that $\mu_{a}=\mu_{A}$, as it follows from the law of iterated expectations. By the previous definition (and decomposition) of the idiosyncratic shock $\theta_{i}$, we observe that the expectations of the following products all agree: $\mathbb{E}_{i}\left[a_{i} \bar{\theta}\right]=\mathbb{E}_{i}\left[A \theta \theta_{i}\right]=\mathbb{E}_{i}[A \bar{\theta}]$. This can be easily seen as follows:

$$
\mathbb{E}\left[\theta_{i} A\right]=\mathbb{E}[\bar{\theta} A]+\mathbb{E}\left[\Delta \theta_{i} A\right]=\mathbb{E}[\bar{\theta} A]+\mathbb{E}[A \cdot \underbrace{\mathbb{E}\left[\Delta \theta_{i} \mid A\right]}_{=0}]=\mathbb{E}[\bar{\theta} A],
$$

where we just use the law of iterated expectations and the fact that the expected value of a idiosyncratic variable conditioned on an aggregate variable must be 0 . Thus:

$$
\operatorname{cov}\left(a_{i}, \bar{\theta}\right)=\operatorname{cov}\left(A, \theta_{i}\right)=\operatorname{cov}(A, \bar{\theta})=\operatorname{cov}\left(a_{i}, \theta_{j}\right)=\mathbb{E}\left[a_{i} \theta_{j}\right]-\mu_{a} \mu_{\theta}=\rho_{a \phi} \sigma_{\theta} \sigma_{a} .
$$

Similarly, since we consider a symmetric Bayes correlated equilibrium, the covariance of the actions of any two individuals, $a_{i}$ and $a_{j}$, which is denoted by $\rho_{a a} \sigma_{a}^{2}$, is equal to the aggregate variance. Once again, this can be easily seen as follows,

$$
\mathbb{E}\left[a_{i} a_{j}\right]=\mathbb{E}\left[A^{2}\right]+\mathbb{E}\left[A \Delta a_{j}\right]+\mathbb{E}\left[\Delta a_{i} A\right]+\mathbb{E}\left[\Delta a_{i} \Delta a_{j}\right]=\mathbb{E}\left[A^{2}\right],
$$

where in this case we need to use that the equilibrium is symmetric and thus $\mathbb{E}\left[\Delta a_{i} \Delta a_{j}\right]=0$. Thus, we have $\sigma_{A}^{2}=\operatorname{cov}\left(a_{i}, a_{j}\right)=\operatorname{cov}\left(A, a_{i}\right)=\rho_{a a} \sigma_{a}^{2}$.

Proof of Proposition 7. The moment equalities (1) and (2) were established in (30) and (31). Thus we proceed to verify that the inequality constraints (3) are necessary and sufficient to guarantee that the matrix $\mathbb{V}$ is positive semidefinite.

Here we express the equilibrium conditions, by a change of variables, in terms of different variables, which facilitates the calculation. Let:

$$
M \triangleq\left(\begin{array}{cccc}
1 & -1 & 0 & 0 \\
0 & 1 & 0 & 0 \\
0 & 0 & 1 & -1 \\
0 & 0 & 0 & 1
\end{array}\right)
$$


Thus, we have that:

$$
\left.\left(\begin{array}{c}
\Delta \theta_{i} \\
\bar{\theta} \\
\Delta a_{i} \\
A
\end{array}\right) \sim \mathcal{N}\left(\left(\begin{array}{c}
0 \\
\mu_{\theta} \\
0 \\
\mu_{a}
\end{array}\right), M \mathbb{V} M^{\prime}\right)\right)
$$

where

$$
\mathbb{V}_{\perp} \triangleq M \mathbb{V} M^{\prime}=\left(\begin{array}{llll}
\left(1-\rho_{\theta \theta}\right) \sigma_{\theta}^{2} & 0 & \left(\rho_{a \theta}-\rho_{a \phi}\right) \sigma_{a} \sigma_{\theta} & 0 \\
0 & \rho_{\theta \theta} \sigma_{\theta}^{2} & 0 & \rho_{a \phi} \sigma_{a} \sigma_{\theta} \\
\left(\rho_{a \theta}-\rho_{a \phi}\right) \sigma_{a} \sigma_{\theta} & 0 & \left(1-\rho_{a a}\right) \sigma_{a}^{2} & 0 \\
0 & \rho_{a \phi} \sigma_{a} \sigma_{\theta} & 0 & \rho_{a a} \sigma_{a}^{2}
\end{array}\right)
$$

We use $\mathbb{V}_{\perp}$ to denote the variance/covariance matrix expressed in terms of $\left(\Delta \theta_{i}, \bar{\theta}, \Delta a_{i}, A\right)$. It is easy to verify that $\mathbb{V}_{\perp}$ is positive semidefinite if and only if the inequality conditions (3) are satisfied. To check this it is sufficient to note that the leading principal minors are positive if and only if these conditions are satisfied, and thus $\mathbb{V}_{\perp}$ is positive semidefinite if and only if these conditions are satisfied.

Proof of Proposition 8. $(\Leftarrow)$ We first prove that if the variables $\left(\theta_{i}, \bar{\theta}, a_{i}, A\right)$ form a Bayes Nash equilibrium for some information structure $\mathcal{I}_{i}$ (and associated signals), then the variables $\left(\theta_{i}, \bar{\theta}, a_{i}, A\right)$ also form a Bayes correlated equilibrium. Consider the case in which agents receive normally distributed signals through the information structure $\mathcal{I}_{i}$, which by minor abuse of notation also serves as conditioning event. Then in any Bayes Nash equilibrium of the game, we have that the actions of the agents are given by:

$$
a_{i}=r \mathbb{E}\left[A \mid \mathcal{I}_{i}\right]+\mathbb{E}\left[\theta_{i} \mid \mathcal{I}_{i}\right], \quad \forall i, \forall \mathcal{I}_{i}
$$

and since the information is normally distributed, the variables $\left(\theta_{i}, \bar{\theta}, a_{i}, A\right)$ are jointly normal as well. By taking the expectation of (50) conditional on the information set $\mathcal{I}_{i}^{\prime}=\left\{\mathcal{I}_{i}, a_{i}\right\}$ we get:

$$
\begin{aligned}
\mathbb{E}\left[a_{i} \mid \mathcal{I}_{i}, a_{i}\right]=a_{i} & =r \mathbb{E}\left[\mathbb{E}\left[A \mid \mathcal{I}_{i}\right] \mid \mathcal{I}_{i}, a_{i}\right]+\mathbb{E}\left[\mathbb{E}\left[\theta_{i} \mid \mathcal{I}_{i}\right] \mid \mathcal{I}_{i}, a_{i}\right] \\
& =r \mathbb{E}\left[A \mid \mathcal{I}_{i}, a_{i}\right]+\mathbb{E}\left[\theta_{i} \mid \mathcal{I}_{i}, a_{i}\right]
\end{aligned}
$$

In other words, agents know the recommended action they are supposed to take, and thus, we can assume that the agents condition on their own actions. By taking expectations of (51) conditional on $\left\{a_{i}\right\}$ we get:

$$
\begin{aligned}
\mathbb{E}\left[a_{i} \mid a_{i}\right]=a_{i} & =r \mathbb{E}\left[\mathbb{E}\left[A \mid \mathcal{I}_{i}, a_{i}\right] \mid a_{i}\right]+\mathbb{E}\left[\mathbb{E}\left[\theta_{i} \mid \mathcal{I}_{i}, a_{i}\right] \mid a_{i}\right] \\
& =r \mathbb{E}\left[A \mid a_{i}\right]+\mathbb{E}\left[\theta_{i} \mid a_{i}\right]
\end{aligned}
$$


where we used the law of iterated expectations. In other words, the information contained in $\left\{a_{i}\right\}$ is a sufficient statistic for agents to compute their best response, and thus the agents compute the same best response if they know $\left\{\mathcal{I}_{i}, a_{i}\right\}$ or if they just know $\left\{a_{i}\right\}$. Yet, looking at (52), by definition $\left(\theta_{i}, \bar{\theta}, a_{i}, A\right)$ form a Bayes correlated equilibrium.

$(\Rightarrow)$ We now prove that if $\left(\theta_{i}, \bar{\theta}, a_{i}, A\right)$ form a Bayes correlated equilibrium, then there exists an information structure $\mathcal{I}_{i}$ such that the variables $\left(\theta_{i}, \bar{\theta}, a_{i}, A\right)$ form a Bayes Nash equilibrium when agents receive this information structure. We consider the case in which the variables $\left(\theta_{i}, \bar{\theta}, a_{i}, A\right)$ form a Bayes correlated equilibrium, and thus the variables are jointly normal and

$$
a_{i}=r \mathbb{E}\left[A \mid a_{i}\right]+\mathbb{E}\left[\theta_{i} \mid a_{i}\right]
$$

Since the variables are jointly normal we can always find $w \in \mathbb{R}$ and $\lambda \in[-1,1]$, such that:

$$
a_{i}=w\left(\lambda \Delta \theta_{i}+(1-|\lambda|) \bar{\theta}+\varepsilon_{i}\right) .
$$

The variables $(\lambda, w)$ and the random variable $\varepsilon$ are defined by the following equations of the Bayes correlated equilibrium distribution:

$$
w \lambda=\frac{\operatorname{cov}\left(a_{i}, \Delta \theta_{i}\right)}{\sigma_{\Delta \theta_{i}}^{2}}, \quad w(1-|\lambda|)=\frac{\operatorname{cov}\left(a_{i}, \bar{\theta}\right)}{\sigma_{\bar{\theta}}^{2}},
$$

and

$$
\varepsilon=\frac{\left.a_{i}-\frac{\operatorname{cov}\left(a_{i}, \Delta \theta_{i}\right) \Delta \theta_{i}}{\sigma_{\Delta \theta_{i}}^{2}}-\frac{\operatorname{cov}\left(a_{i}, \bar{\theta}\right.}{\sigma_{\bar{\theta}}^{2}}\right)}{w} .
$$

Now consider the case in which agents receive a one-dimensional signal

$$
s_{i} \triangleq \frac{a_{i}}{w}=\left(\lambda \Delta \theta_{i}+(1-\lambda) \bar{\theta}+\varepsilon_{i}\right) .
$$

Then, by definition, we have that:

$$
a_{i}=w s_{i}=r \mathbb{E}\left[A \mid a_{i}\right]+\mathbb{E}\left[\theta_{i} \mid a_{i}\right]=r \mathbb{E}\left[A \mid s_{i}\right]+\mathbb{E}\left[\theta_{i} \mid s_{i}\right]
$$

where we use the fact that conditioning on $a_{i}$ is equivalent to conditioning on $s_{i}$. Thus, when agent $i$ receives information structure (and associated signal $s_{i}$ ): $\mathcal{I}_{i}=\left\{s_{i}\right\}$, then agent $i$ taking action $a_{i}=w s_{i}$ constitutes a Bayes Nash equilibrium, as it complies with the best response condition. Thus, the distribution $\left(\theta_{i}, \bar{\theta}, a_{i}, A\right)$ forms a Bayes Nash equilibrium when agents receive signals $\mathcal{I}_{i}=\left\{s_{i}\right\}$. 
Proof of Proposition 9. Note that (54) has the form stated in the Proposition, and thus this was implicitly established by the proof of Proposition 8.

To establish Proposition 12, we use the following lemma that is of independent interest. Consider an arbitrary continuous function:

$$
\psi:[0,1] \times[0,1] \times[-1,1] \rightarrow \mathbb{R}
$$

whose domain is given by the triple of correlation coefficients: $\left(\rho_{a a}, \rho_{a \theta}, \rho_{a \phi}\right)$.

\section{Lemma 2.}

If $\psi\left(\rho_{a a}, \rho_{a \theta}, \rho_{a \phi}\right)$ is a continuous function, strictly increasing in $\rho_{a \theta}$ and weakly increasing in $\rho_{a \phi}$, then the Bayes correlated equilibrium that maximizes $\psi$ is an noise free Bayes correlated equilibrium.

Proof. By rewriting the constraints (34) of Proposition 7 we obtain:

1. $\rho_{\theta \theta} \rho_{a a}-\left(\rho_{a \phi}\right)^{2} \geq 0$;

2. $\left(1-\rho_{a a}\right)\left(1-\rho_{\theta \theta}\right)-\left(\rho_{a \theta}-\rho_{a \phi}\right)^{2} \geq 0$.

If $\psi\left(\rho_{a a}, \rho_{a \theta}, \rho_{a \phi}\right)$ is strictly increasing, then in the optimum the above inequality (2) must bind. Moreover, if the constraint (1) does not bind, then we can just increase $\rho_{a \theta}$ and $\rho_{a \phi}$ in equal amounts, without violating (2) and increasing the value of $\psi$. Thus, in the maximum of $\psi$ we must have that both bind.

Proof of Proposition 10. First, it is easy to see that for any coefficients $\left(\rho_{a a}, \rho_{a \theta}, \rho_{a \phi}\right)$ in the boundary, we must have that that both inequalities in (34) must be satisfied with equality. Otherwise, we could always increase $\rho_{a \theta}$ and $\rho_{a \phi}$ in the right amounts without breaking either inequality, and thus achieving a higher value of $\rho_{a \theta}$. Second, it is easy to solve for $\rho_{a \theta}$ in terms of $\rho_{a a}$ and $\rho_{\theta \theta}$. Using that $\rho_{a \theta} \geq 0$, we must have that:

$$
\rho_{a \theta}=\left|\sqrt{\rho_{a a} \rho_{\theta \theta}} \pm \sqrt{\left(1-\rho_{a a}\right)\left(1-\rho_{\theta \theta}\right)}\right| \text { and } \rho_{a \phi}= \pm \sqrt{\rho_{a a} \rho_{\theta \theta}} .
$$

Finally, it is clear that the boundary will be given by:

$$
\rho_{a \theta}=\sqrt{\rho_{a a} \rho_{\theta \theta}}+\sqrt{\left(1-\rho_{a a}\right)\left(1-\rho_{\theta \theta}\right)},
$$

as this gives the maximum value for $\rho_{a \theta}$.

Proof of Proposition 11. From Proposition 10, we must have that both inequalities in (34) must be satisfied with equality. From (38) it is clear that if both inequalities in (34) are satisfied 
with equality, then $a_{i}$ must be deterministic conditional on $\Delta \theta_{i}$ and $\bar{\theta}$. Moreover, give the properties of multivariate normal distributions, we must have that $a_{i}$ is a linear combination of $\Delta \theta_{i}$ and $\bar{\theta}$. Thus, without loss of generality, for any coefficients $\left(\rho_{a a}, \rho_{a \theta}, \rho_{a \phi}\right)$ in the boundary, we must have that:

$$
a_{i}=w(\lambda)\left((1-|\lambda|) \bar{\theta}+\lambda \Delta \theta_{i}\right) \text { with } \lambda \in[-1,1] .
$$

This comes from the fact that we can calculate the outcome of the Bayes correlated equilibrium as the outcome of a Bayes Nash equilibrium when agents get signals of the form (35), with $\sigma_{\varepsilon}^{2}=0$. For any $\lambda$ such that players action is given by (55) we have that the correlation in actions is given by:

$$
\rho_{a a}=\frac{(1-|\lambda|)^{2} \rho_{\theta \theta}}{(1-|\lambda|)^{2} \rho_{\theta \theta}+\lambda^{2}\left(1-\rho_{\theta \theta}\right)} .
$$

Finally, for any $\lambda \in[0,1]$ we have that the correlation of players action for information structure $-\lambda$ yields the same correlation of action as information structure $\lambda$, but strictly lower correlation between action and payoff state, $\rho_{a \theta}$. Thus, all noise free information structures with $\lambda \in(-1,0)$ cannot be in the boundary as they yield strictly lower $\rho_{a \theta}$ than noise free information structures with $\lambda \in[0,1]$. Thus, all Bayes correlated equilibrium on the boundary form a Bayes Nash equilibrium with a noise free information structure with $\lambda \in[0,1]$.

On the other hand, all Bayes Nash equilibrium with a noise free information structure with $\lambda \in[0,1]$ form a Bayes correlated equilibrium on the boundary. This just come from the fact that actions in Bayes Nash equilibrium with a noise free information structure are linear combination of $\bar{\theta}$ and $\Delta \theta$. Moreover, from (13) and (14) it is easy to see that $\lambda \in[0,1]$ span all correlations satisfying,

$$
\rho_{a \theta}=\sqrt{\rho_{a a} \rho_{\theta \theta}}+\sqrt{\left(1-\rho_{a a}\right)\left(1-\rho_{\theta \theta}\right)}
$$

with $\rho_{a a} \in[0,1]$.

Proof of Proposition 12. From (33) the individual volatility, aggregate volatility and dispersion can be written as follows:

$$
\frac{\rho_{a \theta} \sigma_{\theta}}{1-r \rho_{a a}}, \rho_{a a} \frac{\rho_{a \theta} \sigma_{\theta}}{1-r \rho_{a a}},\left(1-\rho_{a a}\right) \frac{\rho_{a \theta} \sigma_{\theta}}{1-r \rho_{a a}},
$$

and the result follows directly.

Proof of Proposition 13. See supplemental online appendix.

Proof of Proposition 14. See supplemental online appendix. 
[1] Angeletos, G.-M., L. Iovino, and J. LA'O (2011): "Cycles, Gaps, and the Social Value of Information," NBER Working Papers 17229, National Bureau of Economic Research, Inc.

[2] Angeletos, G.-M., And J. LA'O (2009): "Noisy Business Cycles," in NBER Macroeconomics Annual, vol. 24, pp. 319-378. University of Chicago Press.

[3] — (2012): "Optimal Monetary Policy with Informational Frictions," Discussion paper, MIT and University of Chicago.

[4] (2013): "Sentiments," Econometrica, 81, 739-779.

[5] Angeletos, G.-M., and A. Pavan (2007): "Efficient Use of Information and Social Value of Information," Econometrica, 75, 1103-1142.

[6] (2009): "Policy with Dispersed Information," Journal of the European Economic Association, 7, 11-60.

[7] Bergemann, D., T. Heumann, and S. Morris (2014): "Information and Market Power," Yale University and Princeton University.

[8] Bergemann, D., and S. Morris (2013): "Robust Predictions in Games with Incomplete Information," Econometrica, 81, 1251-1308.

[9] - (2014): "The Comparison of Information Structures in Games: Bayes Correlated Equilibrium and Individual Sufficiency," Discussion Paper 1909R, Cowles Foundation for Research in Economics, Yale University.

[10] Clarke, R. (1983): "Collusion and the Incentives for Information Sharing," Bell Journal of Economics, 14, 383-394.

[11] Hellwig, C., and V. Venkateswaran (2009): "Setting the right prices for the wrong reasons," Journal of Monetary Economics, 56, 57 - 77.

[12] Kirby, A. (1988): "Trade Associations as Information Exchange Mechanisms," RAND Journal of Economics, 19, 138-146.

[13] Lorenzoni, G. (2010): "Optimal Monetary Policy with Uncertain Fundamentals and Dispersed Information," Review of Economics Studies, 77, 305-338. 
[14] LuCAS, R. (1972): "Expectations and the Neutrality of Money," Journal of Economic Theory, 4, 103-124.

[15] Mackowiak, B., And M. Wiederholt (2009): "Optimal Sticky Prices under Rational Inattention," American Economic Review, 99(3), 769-803.

[16] Morris, S., And H. Shin (2002): "Social Value of Public Information," American Economic Review, 92, 1521-1534.

[17] NovsheK, W., And H. Sonnenschein (1982): "Fulfilled Expectations Cournot Duopoly with Information Acquisition and Release," Bell Journal of Economics, 13, 214-218.

[18] Raith, M. (1996): "A General Model of Information Sharing in Oligopoly," Journal of Economic Theory, 71, 260-288.

[19] Ui, T., and Y. Yoshizawa (2012): "Radner's Theorem on Teams and Games with a Continuum of Players," Discussion paper, Yokohama National University.

[20] Veldkamp, L. (2011): Information Choice in Macroeconomics and Finance. Princeton University Press.

[21] Venkateswaran, V. (2013): "Heterogeous Information and Labor Market Fluctuations," Discussion paper, New York University.

[22] Vives, X. (1984): "Duopoly Information Equilibrium: Cournot and Bertrand," Journal of Economic Theory, 34, 71-94.

[23] _ (1990): "Trade Association Disclosure Rules, Incentives to share information, and welfare," RAND Journal of Economics, 21, 409-430.

[24] (1999): Oligopoly Pricing. MIT Press, Cambridge.

[25] _ (2014): "On the Possibility of Informationally Efficient Markets," Journal of the European Economic Association, forthcoming. 